\title{
Shifts in podocyte histone H3K27me3 regulate mouse and human glomerular disease
}

\author{
Syamantak Majumder, ${ }^{1}$ Karina Thieme,, ${ }^{1}$ Sri N. Batchu, ${ }^{1}$ Tamadher A. Alghamdi,, Bridgit B. Bowskill, ${ }^{1}$ M. Golam Kabir, ${ }^{1}$ \\ Youan Liu, ${ }^{1}$ Suzanne L. Advani, ${ }^{1}$ Kathryn E. White, ${ }^{2}$ Laurette Geldenhuys, ${ }^{3}$ Karthik K. Tennankore, ${ }^{4}$ Penelope Poyah, ${ }^{4}$ \\ Ferhan S. Siddiqi, ${ }^{4}$ and Andrew Advani ${ }^{1}$ \\ ${ }^{1}$ Keenan Research Centre for Biomedical Science and Li Ka Shing Knowledge Institute of St. Michael's Hospital, Toronto, Ontario, Canada. ${ }^{2}$ Electron Microscopy Research Services, Newcastle University, \\ Newcastle upon Tyne, United Kingdom. ${ }^{3}$ Department of Pathology and ${ }^{4}$ Department of Medicine, Dalhousie University, Halifax, Nova Scotia, Canada.
}

\begin{abstract}
Histone protein modifications control fate determination during normal development and dedifferentiation during disease. Here, we set out to determine the extent to which dynamic changes to histones affect the differentiated phenotype of ordinarily quiescent adult glomerular podocytes. To do this, we examined the consequences of shifting the balance of the repressive histone $\mathrm{H} 3$ lysine 27 trimethylation (H3K27me3) mark in podocytes. Adriamycin nephrotoxicity and subtotal nephrectomy (SNx) studies indicated that deletion of the histone methylating enzyme EZH2 from podocytes decreased H3K27me3 levels and sensitized mice to glomerular disease. H3K27me3 was enriched at the promoter region of the Notch ligand Jag1 in podocytes, and derepression of Jag1 by EZH2 inhibition or knockdown facilitated podocyte dedifferentiation. Conversely, inhibition of the Jumonji C domain-containing demethylases Jmjd3 and UTX increased the H3K27me3 content of podocytes and attenuated glomerular disease in adriamycin nephrotoxicity, SNx, and diabetes. Podocytes in glomeruli from humans with focal segmental glomerulosclerosis or diabetic nephropathy exhibited diminished H3K27me3 and heightened UTX content. Analogous to human disease, inhibition of Jmjd3 and UTX abated nephropathy progression in mice with established glomerular injury and reduced H3K27me3 levels. Together, these findings indicate that ostensibly stable chromatin modifications can be dynamically regulated in quiescent cells and that epigenetic reprogramming can improve outcomes in glomerular disease by repressing the reactivation of developmental pathways.
\end{abstract}

\section{Introduction}

The glomerulus is the basic filtering unit of the kidney. Its sieving action is made possible by highly specialized cells, termed podocytes, that reside within it. Arising from the metanephric mesenchyme during organogenesis, podocytes are highly differentiated epithelial cells that wrap their interlocking pedicels around the glomerular capillaries. When podocytes lose their differentiated architecture, plasma proteins are wasted in the urine. Podocyte damage is a common feature of many glomerular diseases that are collectively responsible for over three-quarters of all cases of chronic kidney disease (CKD) in the US (1). For instance, podocyte dysfunction underlies the development of focal segmental glomerulosclerosis (FSGS), the most common cause of idiopathic nephrotic syndrome (2), and it also contributes to the development of diabetic nephropathy, the most common cause of kidney failure across the globe (3).

The differentiated fate of each new cell is determined and maintained by chromatin modifications (4). Trimethylation of lysine residue 27 on histone protein $\mathrm{H} 3$ (H3K27me3), for exam-

Authorship note: S. Majumder and K. Thieme contributed equally to this work. Conflict of interest: A. Advani has received research support from Boehringer Ingelheim and AstraZeneca and is listed as an inventor on a patent application (WO 2015/128453) submitted by Boehringer Ingelheim.

Submitted: June 29, 2017; Accepted: October 31, 2017.

Reference information: / Clin Invest. 2018;128(1):483-499.

https://doi.org/10.1172/JCI95946. ple, represses the expression of nearby genes that encode proteins typically associated with alternative cellular lineages (5). Gain of the H3K27me3 mark is catalyzed by the histone methyltransferase enzyme, enhancer of zeste homolog 2 (EZH2), the catalytic subunit of polycomb repressive complex 2 (PRC2) (6), and was once considered to be a stable and irreversible process (7). However, within the past decade, the discovery of histone lysine-specific demethylating enzymes has challenged this dogma (7). In the case of H3K27me3, the histone mark can be erased by 2 demethylases belonging to the Jumonji C (JmjC) domain-containing family:ubiquitously transcribed tetratricopeptide repeat on chromosome $\mathrm{X}$ (UTX, also called lysine-specific demethylase 6A [KDM6A]) and Jumonji D3 (Jmjd3, also called KDM6B) (8-11). The JmjC domaincontaining proteins are the most recently discovered and largest class of histone demethylases. They catalyze histone lysine demethylation through $\mathrm{Fe}(\mathrm{II})$ - and $\alpha$-ketoglutarate-dependent hydroxylation or dioxygenase reactions (12). UTX and Jmjd3 share $84 \%$ sequence similarity in their JmjC catalytic domains (13), and both enzymes possess a zinc-binding domain that provides specificity for H3K27 over other methylated histone lysine residues (14, 15). We recently discovered that systemic EZH2 inhibition accelerates kidney injury in diabetes (16). However, except in the cancer setting, the actions of JmjC demethylases in the kidney have not previously been described.

In dedifferentiated cancer cells, reexpression of histonemodifying enzymes is often associated with reversion to an earlier developmental stage (17). Like cancer, podocyte damage in 
A Nephrin H3K27me3

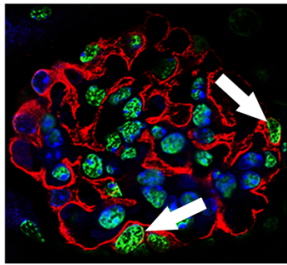

C

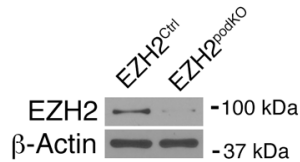

E

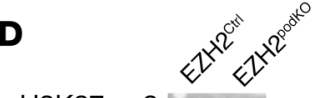

H3K27me3 - $-15 \mathrm{kDa}$ Histone $\mathrm{H3}--15 \mathrm{kDa}$

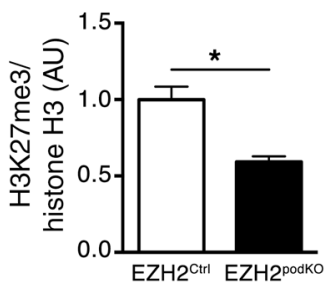

G
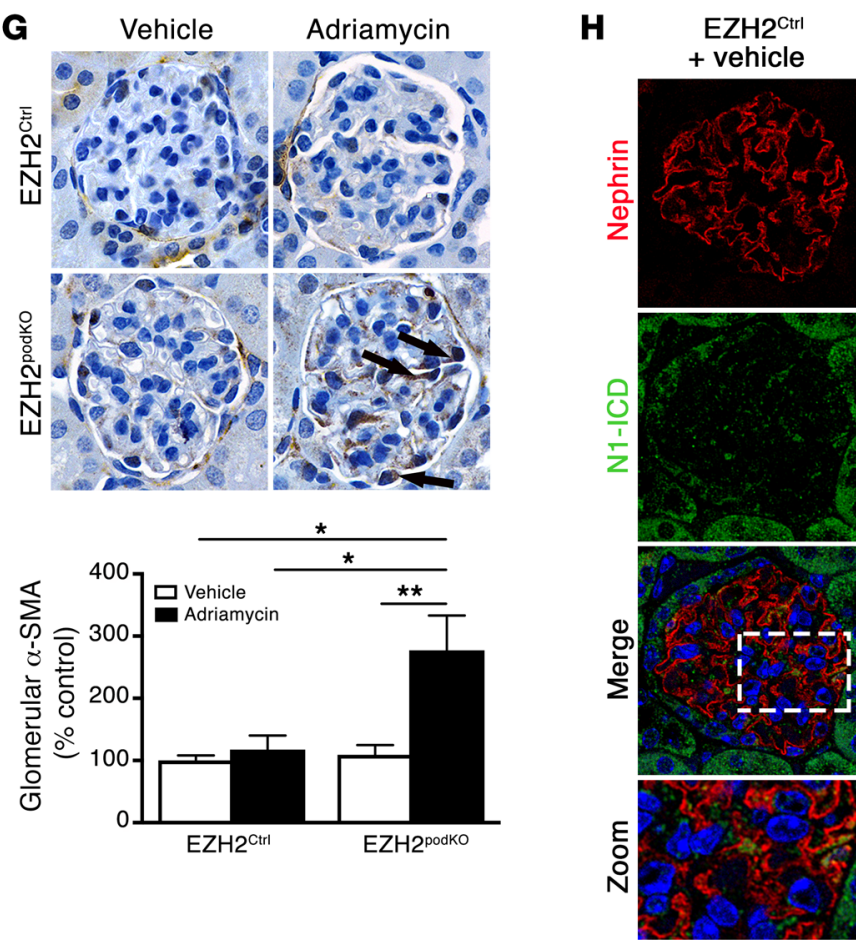

B
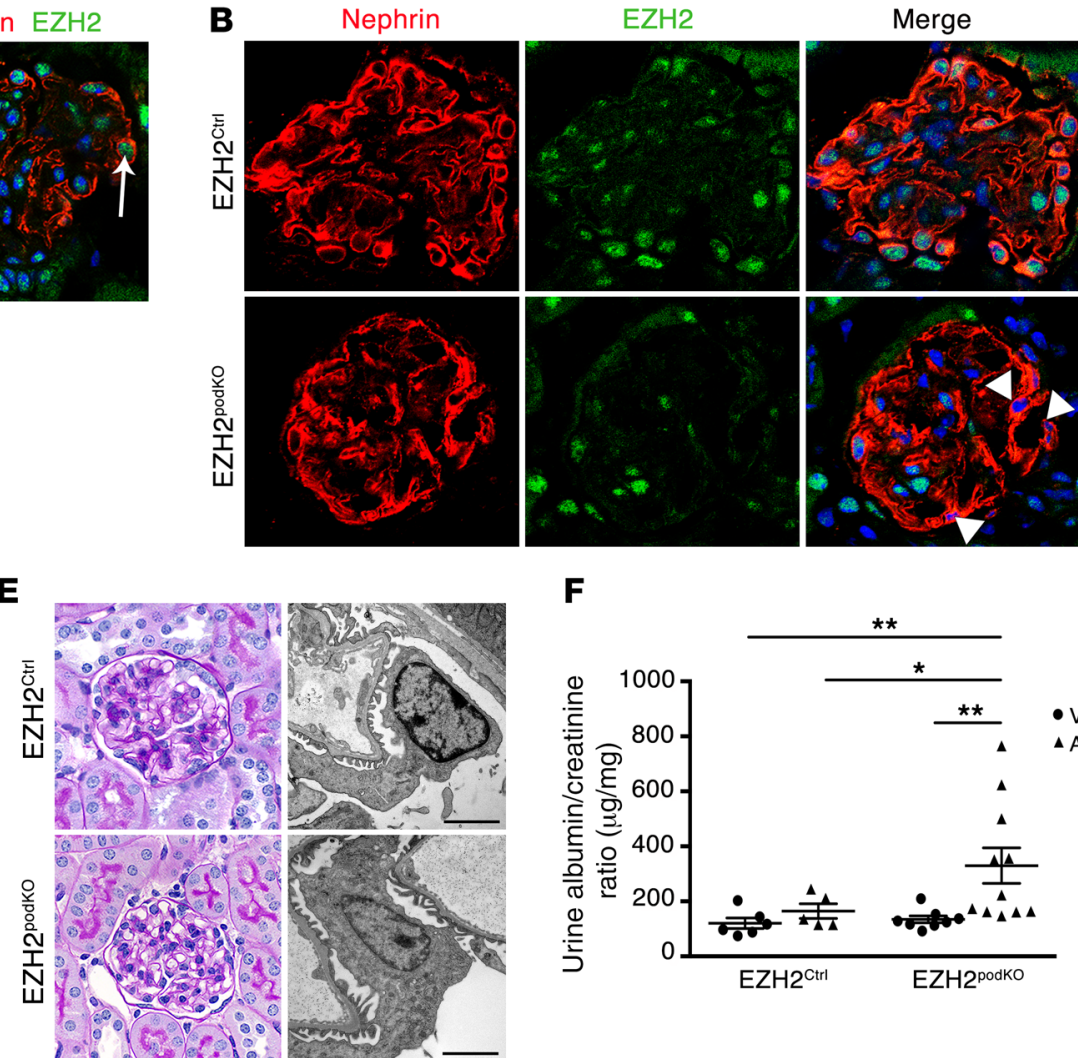

F

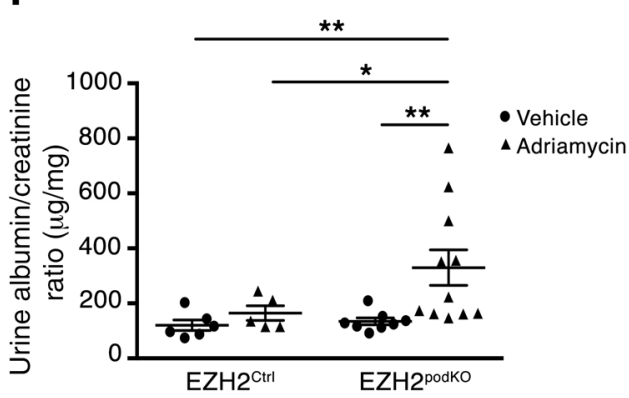

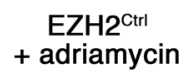

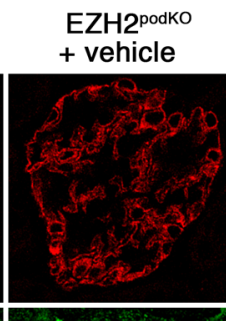

EZH2podko + adriamycin
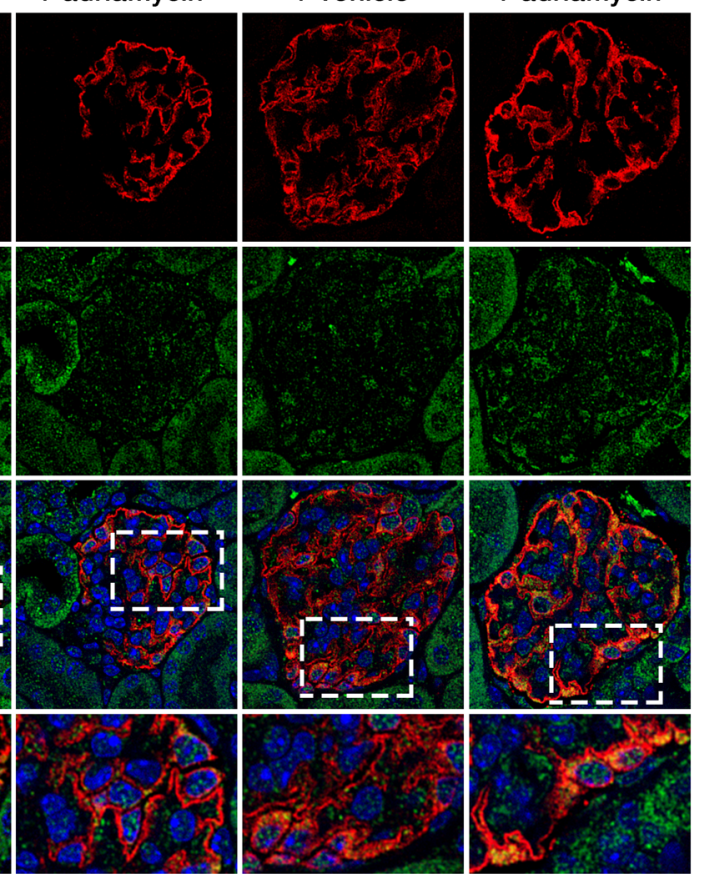
Figure 1. Decreasing H3K27me3 levels in podocytes renders mice susceptible to glomerular injury. (A) Dual immunofluorescence of normal mouse glomeruli stained for $\mathrm{H} 3 \mathrm{~K} 27 \mathrm{me} 3(n=3)$ or $\mathrm{EZH} 2(n=3)$ and the podocyte protein nephrin. DAPI staining is shown in blue. Thick white arrows label H3K27me3 in mouse podocytes, and thin white arrows label EZH2 in mouse podocytes. (B) Dual immunofluorescence for nephrin and EZH2 in glomeruli from EZH $2^{\mathrm{Ctrl}}(n=3)$ and $\mathrm{EZH}^{\text {podko }}(n=3)$ mice showing diminished podocyte $\mathrm{EZH2}$ in the EZH2 ${ }^{\text {podko }}$ glomerulus (arrowheads). (C) Immunoblotting for EZH2 in primary cultured podocytes from EZH2 $2^{\mathrm{Ctrl}}(n=$ $3)$ and EZH2 $2^{\text {podKo }}(n=3)$ mice. (D) Immunoblotting for H3K27me3 in primary cultured podocytes from EZH2 $2^{\text {ctrl }}(n=3)$ and EZH2 ${ }^{\text {podko }}(n=3)$ mice. (E) PASstained kidney sections (original magnification, $\times 400$ ) and transmission electron micrographs from EZH2 ${ }^{\mathrm{Ctrl}}(n=3)$ and $\mathrm{EZH}^{\text {podko }}(n=3)$ mice under basal conditions. Scale bars: $2 \mu \mathrm{m}$. (F) Urine albumin/creatinine ratio 10 days after injection of adriamycin into $\mathrm{EZH}^{\mathrm{ctrl}}$ (vehicle, $n=6$; adriamycin, $n=5$ ) and EZH2 ${ }^{\text {podko }}$ (vehicle, $n=8$; adriamycin, $n=11$ ) mice. (C) Immunostaining for $\alpha$-SMA in EZH2 $2^{\text {Ctrl }}$ (vehicle, $n=3$; adriamycin, $n=4$ ) and EZH2 $2^{\text {podko }}$ (vehicle, $n=7$; adriamycin, $n=8$ ) mice 10 days after adriamycin injection. Original magnification, $\times 400$. Black arrows label $\alpha$-SMA immunoreactivity in glomerular cells of the adriamycin-injected $\mathrm{EZH} 2^{\text {podko }}$ mouse. (H) Dual immunofluorescence for nephrin and the truncated Notch receptor $\mathrm{N} 1-\mathrm{ICD}$ in $\mathrm{EZH}_{2}{ }^{\mathrm{Ctrl}}$ (vehicle, $n=3$; adriamycin, $n=3$ ) and $\mathrm{EZH} 2^{\text {podko }}$ (vehicle, $n=3$; adriamycin, $n=3$ ) mice 10 days after adriamycin injection. DAPI staining is shown in blue. Zoomed images are enlargements of the outlined areas. Original magnification, $\times 630$. Values represent the mean \pm SEM. ${ }^{*} P<0.05$ and ${ }^{* *} P<0.01$, by 2 -tailed Student's $t$ test (D) and 1-way ANOVA followed by Fisher's LSD post-hoc test ( $\mathbf{F}$ and $\mathbf{G}$ ).

glomerular disease is also associated with the reactivation of developmental pathways (18). However, unlike cancer cells, podocytes are postmitotic. As such, their dedifferentiation provides a useful means to gauge the extent to which dynamic shifts may occur in apparently stable epigenetic marks in apparently quiescent cells. On this background, we hypothesized that alterations in H3K27me3 levels affect the reactivation of developmental pathways in podocytes and that this in turn alters the natural history of glomerular disease.

\section{Results}

Genetic removal of EZH 2 from podocytes decreases $H 3 K 27 m e 3$ levels and sensitizes mice to glomerular disease. In an initial histochemical survey, we observed high levels of both H3K27me3 and the H3K27 trimethylating enzyme EZH2 in podocytes from mouse kidneys (Figure 1A). We wondered whether removing EZH2 from podocytes would diminish H3K27me3 levels and whether this would have any effect on the development of glomerular disease. Accordingly, we generated mice with podocyte-specific knockout of EZH2. To do this, we mated podocin-Cre ${ }^{+}$mice that express Cre recombinase in podocytes under the control of the human podocin $N P H S 2$ promoter (19) with $E z h 2^{f / / l}$ mice that were generated by the introduction of LoxP sites into the $E z h 2$ locus flanking exons 16-19, which encode the essential SET domain of the protein (20). We studied 2 groups of mice: podocin-Cre $\mathrm{Ezh}^{+/ /+}$and podocin-Cre ${ }^{+}$ $E z h 2^{f / f l}$ mice, hereafter referred to as EZH2 $2^{\mathrm{Ctrl}}$ and $\mathrm{EZH} 2^{\text {podKo }}$ mice, respectively. EZH $2^{\text {podKo }}$ mice were born at the expected Mendelian frequency. Excision of EZH2 from podocytes of EZH2podko mice was confirmed by immunofluorescence staining of kidney sections (Figure 1B) and by immunoblotting primary cultured podocytes (Figure 1C) and was accompanied by an approximately $50 \%$ reduction in podocyte H3K27me3 content (Figure 1D). Despite this reduction in podocyte H3K27me3 content, glomerular structure and podocyte ultrastructure appeared unaffected in EZH2 $2^{\text {podko }}$ mice under normal conditions (Figure 1E).

To determine whether the diminution in podocyte H3K27me3 content would sensitize EZH2 $2^{\text {podKo }}$ mice to glomerular disease, we exposed the mice to the podocyte toxin adriamycin, which is commonly used to mimic FSGS-like glomerular injury (21). Consistent with their predominant adriamycin-resistant C57BL/6-strain background $(22,23)$, we found that albuminuria was unaffected by adriamycin in $\mathrm{EZH} 2^{\mathrm{Ctrl}}$ mice (Figure 1F), whereas it was increased in EZH2 $2^{\text {podKo }}$ mice after adriamycin injection (Figure 1F). Likewise, both glomerular expression of the mesenchymal marker $\alpha$-smooth muscle actin ( $\alpha$-SMA) (Figure 1G) and mesangial matrix accumulation (Supplemental Figure 1; supplemental material available online with this article; https://doi.org/10.1172/JCI95946DS1) were increased in adriamycin-exposed EZH2 $2^{\text {podKo }}$ mice, whereas they were unaltered in adriamycin-exposed $\mathrm{EZH} 2^{\text {ctrl }}$ mice and in untreated EZH2 $2^{\text {podKo }}$ mice (Figure $1 G$ and Supplemental Figure 1). Because reactivation of the Notch pathway has been linked to podocyte dedifferentiation during the development of glomerular disease $(24,25)$, we probed for glomerular Notch pathway activity. In so doing, we observed that podocytes of adriamycin-injected EZH2 ${ }^{\text {podKo }}$ mice exhibited heightened levels of N1-ICD (Figure $1 \mathrm{H}$ and Supplemental Figure 2A), the active truncated form of the receptor Notch1, which is generated following juxtacrine ligandinitiated receptor cleavage, as well as increased expression of the Notch target gene Hes-related repressor protein 1 (Hey1) (Supplemental Figure 2B). In contrast, we found that podocyte density (transmission electron microscopy) and numbers (WT1 immunostaining) were unaffected in adriamycin-injected EZH2 $2^{\text {podko }}$ mice (Supplemental Figure 3, A and B). We were unable to detect podocyte apoptosis in these mice by immunofluorescence microscopy for caspase-3 cleavage or by transmission electron microscopy, whereas glomerular cell TUNEL staining was unaltered across the study groups (Supplemental Figure 3, C and D). To determine whether the susceptibility of EZH2 $2^{\text {podKo }}$ mice to glomerular disease was specific for adriamycin toxicity, we subjected mice to resection of approximately five-sixths of the kidney mass (subtotal nephrectomy [SNx]), a surgical model of FSGS-like disease (26). After 6 weeks, albuminuria tended to be higher in SNxoperated EZH $2^{\text {Ctrl }}$ mice than in their sham-operated counterparts, whereas it was increased by approximately 4 -fold in SNx-operated EZH2 ${ }^{\text {podko }}$ mice (Supplemental Figure 4A) and accompanied by heightened podocyte N1-ICD levels (Supplemental Figure 4B).

Loss of H3K27me3 upregulates Jagged-1 in podocytes and promotes podocyte dedifferentiation. To determine how loss of the H3K27me3 mark from podocytes activates the Notch pathway, we exposed cultured, immortalized mouse podocytes to the inhibitor of EZH2 enzymatic activity EPZ-6438 ( $\mathrm{IC}_{50}$ of $9 \mathrm{nM}$ for EZH2 vs. $392 \mathrm{nM}$ for EZH1 and >50,000 nM for 14 other histone methyltransferases; ref. 27). Before doing this, we administered EPZ-6438 to adriamycin-sensitive BALB/c mice and observed an increase in albuminuria with the EZH2 inhibitor following adriamycin injection (Supplemental Figure 5), which paralleled the increase in albuminuria we had seen in adriamycin-treated EZH2 ${ }^{\text {podKo }}$ mice (Figure 1F). In cultured mouse podocytes, EZH2 inhibition with EPZ-6438 led to a decrease in H3K27me3 levels 


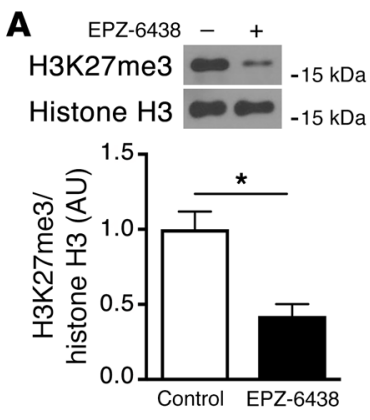

E Notch1 siRNA - +

Notch1 (NTM) $--100 \mathrm{kDa}$ $\beta$-Actin $=-37 \mathrm{kDa}$
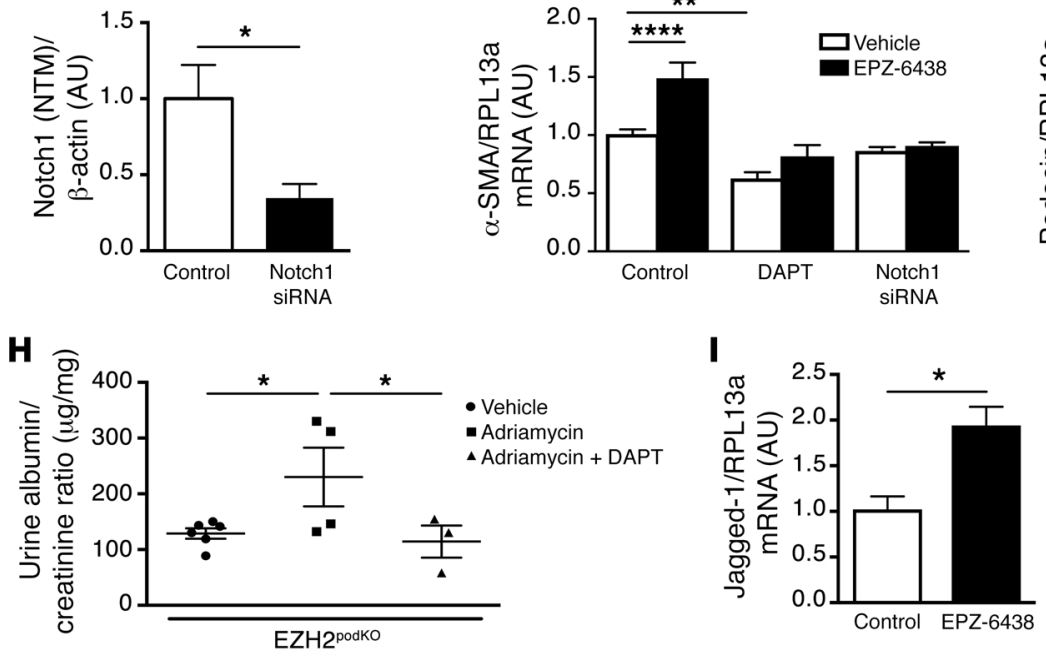

$\mathbf{F}$

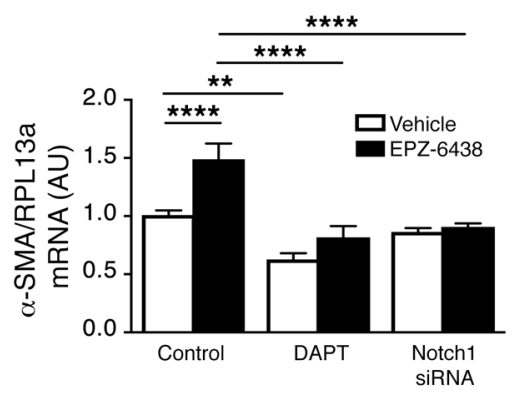

$\mathbf{G}$
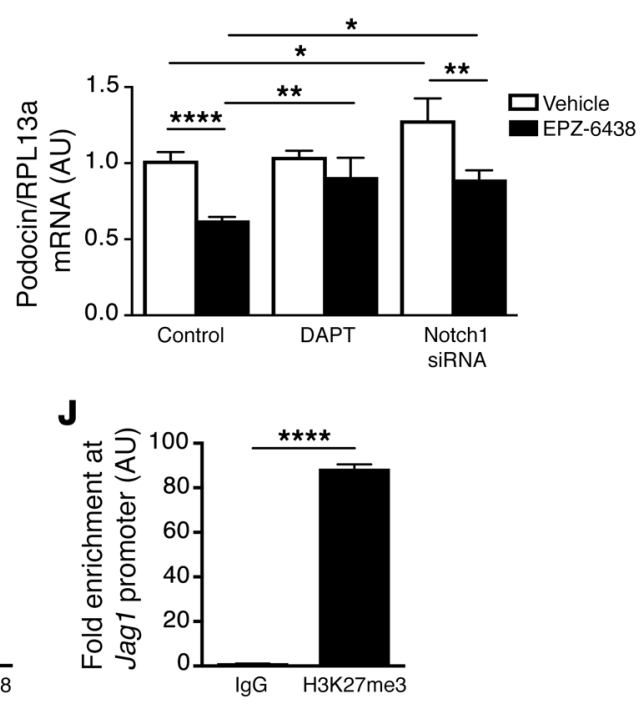
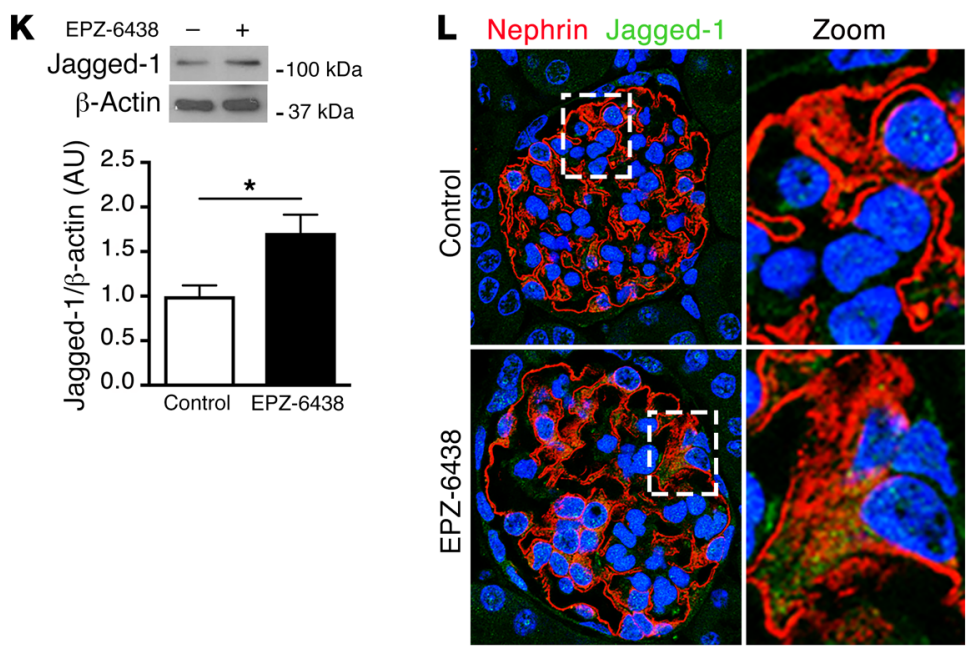

Figure 2. Decreasing H3K27me3 levels promotes Notch-dependent podocyte dedifferentiation. (A and B) Immunoblotting for H3K27me3 $(n=3)(\mathbf{A})$ and N1-ICD $(n=3)(B)$ in mouse podocytes exposed to $10 \mu \mathrm{M}$ EPZ-6438 for 48 hours. (C) Immunofluorescence to detect N1-ICD in mouse podocytes treated with EPZ-6438, showing increased nuclear N1-ICD expression following EPZ-6438 treatment $(n=3)$. Original magnification, $\times 630$. (D) mRNA levels of the Notch target gene Hey1 following EPZ-6438 treatment $(n=3)$. (E) Immunoblotting of cultured mouse podocytes for the cleaved NTM of Notch1 after knockdown of Notch1 with siRNA $(n=5)$. ( $\mathbf{F}$ and $\mathbf{G})$ mRNA levels of the mesenchymal marker $\alpha$-SMA (F) and the podocyte marker podocin (G) in podocytes treated with EPZ-6438 for 72 hours $(n=12)$, with or without pretreatment with the $\gamma$ secretase inhibitor DAPT (10 $\mu \mathrm{M}, n=6)$ or knockdown of Notch1 with siRNA $(n=6)$. (H) Urine albumin/creatinine ratio in EZH2 $2^{\text {podko }}$ $(n=6)$ mice injected with adriamycin $(n=4)$ and treated with DAPT ( $20 \mathrm{mg} / \mathrm{kg}$ i.p. every 3 days) $(n=3)$ for 10 days. (I) Jagged-1 mRNA levels in mouse podocytes treated with EPZ-6438 $(n=3)$. (J) ChIP for the Jag1 promoter following enrichment with an H3K27me3 antibody $(n=3)$. (K) Immunoblotting for Jagged-1 in mouse podocytes treated with EPZ-6438 $(n=3)$. (L) Immunofluorescence to detect the podocyte marker nephrin and Jagged-1 in glomeruli from a BALB/c mouse and from a BALB/c mouse treated with EPZ-6438 $(100 \mathrm{mg} / \mathrm{kg}$ by daily gavage for 10 days) (vehicle, $n=5$; EPZ-6438, $n=6$ ). DAPI staining is shown in blue. Zoomed images are enlargements of the outlined areas. Original magnification, $\times 630$. Values represent the mean \pm SEM. ${ }^{*} P<0.05$, ${ }^{* *} P<0.01$, and ${ }^{* * *} P<0.0001$, by 2-tailed Student's $t$ test (A, B, D, E, I, J, and $\mathbf{K}$ ) and 1-way ANOVA followed by Fisher's LSD post-hoc test $(\mathbf{F}-\mathbf{H})$. 
(Figure 2A) that was also accompanied by (a) increased cellular content of N1-ICD (Figure 2B); (b) increased nuclear N1-ICD levels (Figure 2C); and (c) increased expression of the Notch target gene Hey1 (Figure 2D). To determine whether EZH2 inhibition with EPZ-6438 affects podocyte dedifferentiation in a Notchdependent manner, we treated podocytes with either the $\gamma$ secretase inhibitor $\mathrm{N}$-[N-(3,5-difluorophenacetyl)-l-alanyl]-S-phenylglycine t-butyl ester (DAPT), which blocks Notch receptor cleavage at the cell surface (28), or with sequence-specific siRNA targeting the Notch receptor Notch1 (Figure 2E). We found that treatment with EPZ-6438 altered the expression of markers of podocyte dedifferentiation, which was reflected by increased expression of the mesenchymal marker $\alpha$-SMA (Figure $2 F$ ) and decreased expression of the podocyte differentiation marker podocin (Figure $2 \mathrm{G})$, whereas either DAPT or Notch1 siRNA negated these effects (Figure 2, F and G). Similarly, treatment of EZH2 ${ }^{\text {podKo }}$ mice with DAPT abolished adriamycin-induced albuminuria (Figure $2 \mathrm{H}$ ).

Next, we questioned how H3K27me3 normally restrains Notch pathway activity. We recognized the previously described association of the Notch receptors Notch1 and Notch2 and the Notch ligand Jagged-1 with proteinuric nephropathy in patients (29), and we performed an initial screen for mRNA changes in these candidates in EPZ-6438-exposed podocytes. With this approach, we observed an approximate doubling of mRNA levels of the Notch ligand Jagged-1 with H3K27me3 depletion (Figure 2I), whereas Notch1 and Notch2 mRNA levels were unaltered (fold change with EPZ-6438: Notch1, $0.9 \pm 0.1$; Notch2, $1.0 \pm 0.1$ ). To determine whether H3K27me3 is associated with the Jagged-1 (Jag1) promoter, we performed ChIP and detected a more than 80 -fold enrichment of H3K27me3 at the Jag1 promoter (Figure $2 \mathrm{~J})$. We observed an increase in Jagged-1 protein levels in EPZ6438-treated cultured podocytes (Figure 2K) and in podocytes of EPZ-6438-treated mice (Figure 2L). Finally, we found that decreasing EZH2 levels with shRNA (Supplemental Figure 6A) had effects similar to those of EZH2 inhibition with EPZ-6438, causing a decrease in podocyte H3K27me3 levels (Supplemental Figure 6B) that was accompanied by increased Notch pathway activation (increased Jagged-1 [Supplemental Figure 6C] and N1-ICD [Supplemental Figure 6D]) and podocyte dedifferentiation (increased $\alpha$-SMA [Supplemental Figure 6E] and decreased podocin [Supplemental Figure 6F] mRNA levels).

Inhibition of the JmjC domain-containing proteins Jmjd3 and UTX increases podocyte H3K27me 3 content and restrains Notch pathway activity. Having found that decreasing podocyte H3K27me3 levels promotes podocyte dedifferentiation and glomerular disease, we wondered whether increasing H3K27me3 levels would prevent podocyte dedifferentiation and glomerular disease. To explore this possibility, we took advantage of a recently developed selective inhibitor of Jmjd3 and UTX, GSK-J4 (the cell-permeable prodrug of GSK-J1, with an $\mathrm{IC}_{50}$ of $60 \mathrm{nM}$ in a cell-free assay; ref. 30), which we found to cause a more than 3-fold increase in mouse podocyte H3K27me 3 content (Figure 3A). Coincident with the increase in $\mathrm{H} 3 \mathrm{~K} 27 \mathrm{me} 3$ levels in cultured podocytes, both Jagged-1 mRNA (Figure 3B) and Jagged-1 protein (Figure 3C) levels were reduced with GSK-J4 treatment. Correspondingly, when we exposed podocytes to the inducer of dedifferentiation TGF- $\beta 1$ (31), we found that pretreatment with GSK-J4 prevented both the increase in intracellular N1-ICD levels (Figure 3D) and the increase in $\alpha$-SMA (Figure $3 \mathrm{E}$ ) and the decrease in podocin (Figure $3 F$ ) mRNA levels. Cognizant of a recent report indicating that GSK-J4 may also affect the activity of the H3K4 demethylases Jarid1B/C (32), we immunoblotted podocyte lysates for H3K4me1 and H3K4me3 and observed no change in these chromatin marks with GSK-J4 treatment (Supplemental Figure 7, A and B). Furthermore, when we knocked down Jmjd3 and UTX with siRNA (Supplemental Figure 7, C and D), we observed a reduction in podocyte Jagged-1 levels (Supplemental Figure 7E) similar to that seen with GSK-J4 treatment (Figure 3C). Next, to determine whether increasing H3K27me3 levels with Jmjd 3 and UTX inhibition attenuates glomerular disease, we treated adriamycin-injected BALB/c mice with GSK-J4. Initial experiments in normal BALB/c mice revealed that administration of GSK-J4 at a dose of $10 \mathrm{mg} / \mathrm{kg}$ by thrice-weekly i.p. injections led to an increase of approximately 4-fold in renal H3K27me3 content (Figure 3G), with an increase in podocyte $\mathrm{H} 3 \mathrm{~K} 27 \mathrm{me} 3$ levels detected by dual immunofluorescence microscopy (Figure 3H). Moreover, GSK-J4 treatment markedly attenuated the rise in albuminuria with adriamycin treatment (Figure 3I) and limited both glomerular Jagged-1 upregulation and loss of the podocyte protein nephrin (Supplemental Figure 8).

Inhibition of Jmjd3 and UTX attenuates the development of kidney disease in diabetic mice. Given the renoprotective effect of Jmjd3 and UTX inhibition in adriamycin-injected mice and cognizant that the most common cause of kidney failure is diabetes, we next queried whether GSK-J4 treatment may attenuate the development of glomerular injury in diabetic mice. To address this question, we elected to study $\mathrm{db} / \mathrm{db}$ mice (an obese model of type 2 diabetes) and their nondiabetic, nonobese $\mathrm{db} / \mathrm{m}$ counterparts. Diabetes was confirmed in $\mathrm{db} / \mathrm{db}$ mice at 8 weeks of age, and the animals were then randomized to receive GSK-J4 or vehicle by thrice-weekly i.p. injection for a further 10 weeks. We found that body weight (Figure $4 \mathrm{~A}$ ) and kidney weight (Figure $4 \mathrm{~B}$ ) were both increased in $\mathrm{db} / \mathrm{db}$ mice in comparison with $\mathrm{db} / \mathrm{m}$ mice and that neither parameter was affected by GSK-J4 treatment. Blood glucose levels were marginally increased with GSK-J4 in $\mathrm{db} / \mathrm{m}$ mice and marginally decreased with GSK-J4 in db/db mice (Figure 4C). However, glycated hemoglobin levels were unchanged in $\mathrm{db} / \mathrm{m}$ mice and unexpectedly slightly increased with GSK-J4 in db/db mice (Figure 4D). Despite these subtle changes in dysglycemia, we found that GSK-J4 had no effect on urine flow in $\mathrm{db} / \mathrm{db}$ mice (Figure $4 \mathrm{E}$ ), although both albuminuria (Figure $4 \mathrm{~F}$ ) and the accumulation of matrix within the glomerular mesangium (Supplemental Figure 9A) were diminished with GSK-J4. Similarly, podocyte foot process effacement (Figure 4G) and glomerular $\alpha$-SMA (Supplemental Figure 9B) and Jagged-1 (Figure 4H) upregulation in $\mathrm{db} / \mathrm{db}$ mice were each attenuated with GSK-J4 treatment. We probed for podocyte loss and podocyte programmed cell death using several complementary approaches, as we had done in adriamycin-exposed EZH2 $2^{\text {podko }}$ mice. Both podocyte density, determined by transmission electron microscopy (Supplemental Figure 10A), and podocyte numbers, determined by WT1 immunostaining (Supplemental Figure 10B), were reduced in $\mathrm{db} / \mathrm{db}$ mice in comparison with $\mathrm{db} / \mathrm{m}$ mice. However, neither parameter was affected by GSK-J4 treatment (Supplemental Figure 10, A and B). In these experiments and the time point studied (age 18 weeks), glomerular TUNEL staining was 
A
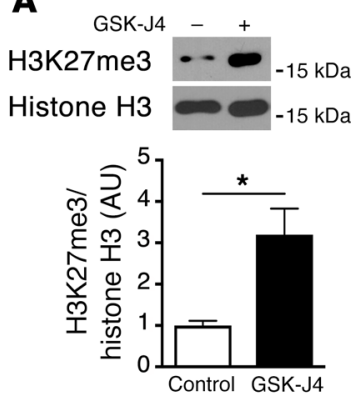

B

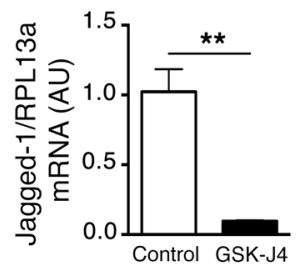

C
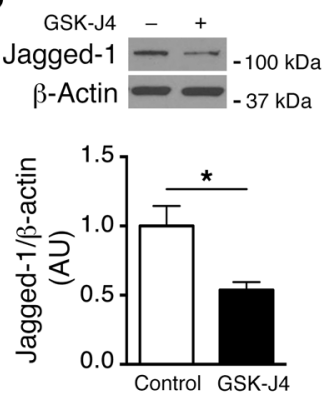

D

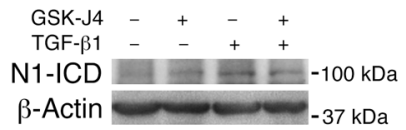

$\beta$-Actin $-37 \mathrm{kDa}$

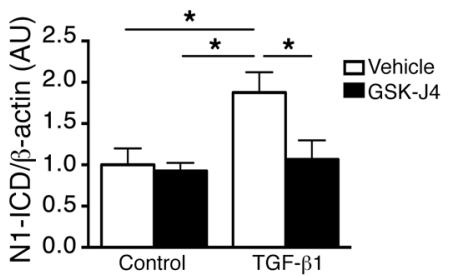

G

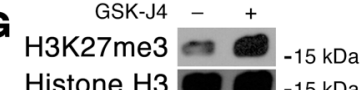

Histone H3 -15 kDa

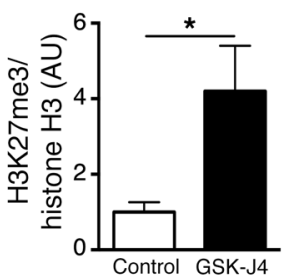

H

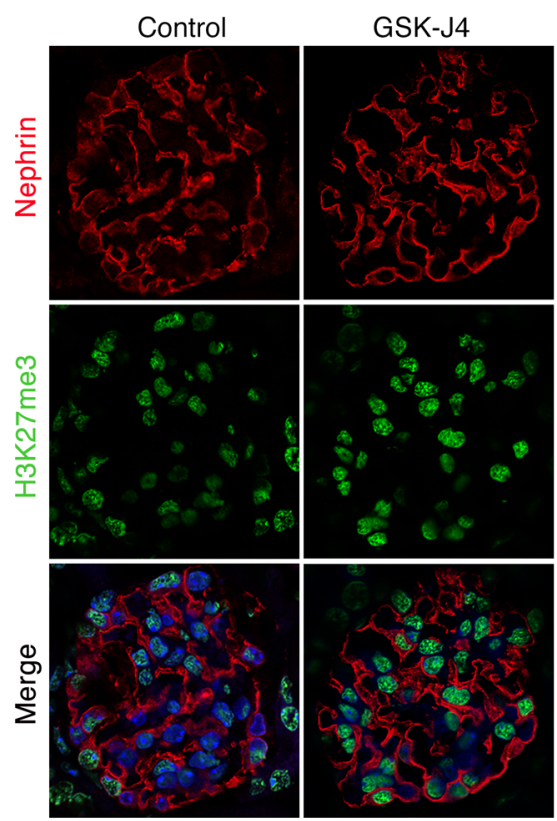

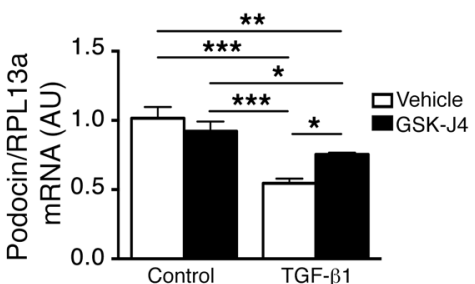

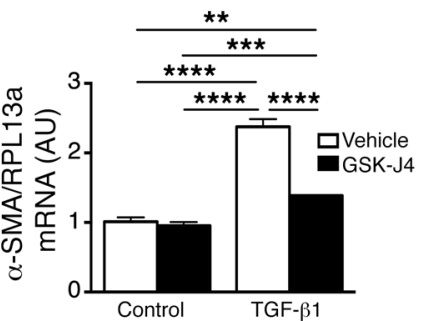

$\mathbf{F}$

I

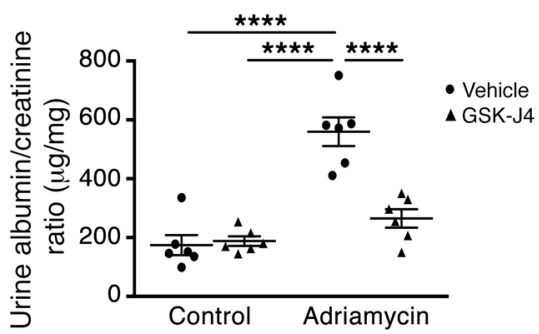

Figure 3. Inhibition of Jmjd3 and UTX increases podocyte H3K27me3 levels and attenuates Notch-mediated dedifferentiation. (A) Immunoblotting for H3K27me3 in cultured mouse podocytes treated with the Jmjd3 and UTX inhibitor GSK-J4 (5 $\mu$ M for 48 hours) $(n=3)$. (B and C) Effect of GSK-J4 on podocyte Jagged-1 mRNA ( $n=3$ ) (B) and protein ( $n=5)$ (C) levels. (D) N1-ICD levels in mouse podocytes exposed to recombinant TCF- $\beta 1(10 \mathrm{ng} / \mathrm{ml}$ for 48 hours) in the presence or absence of GSK-J4 $(5 \mu \mathrm{M})(n=3)$. (E and F) $\alpha$-SMA $(\mathbf{E})(n=6)$ and podocin $(\mathbf{F})(n=6)$ mRNA levels in podocytes exposed to TGF- $\beta 1$, with or without GSK-J4. (C) Immunoblotting for H3K27me3 in kidney homogenates of mice treated with GSK-J4 (10 mg/kg thrice weekly i.p. for 10 days) $(n=3)$. (H) Immunofluorescence to detect the podocyte marker nephrin and H3K27me3 in glomeruli from mice treated with vehicle or GSK-J4 for 10 days, showing increased nuclear H3K27me3 levels in nephrin-positive glomerular cells $(n=3)$. DAPI staining is shown in blue. Original magnification, $\times 630$. (I) Urine albumin/creatinine ratio in BALB/c mice injected with adriamycin or vehicle and treated with GSK-J4 $10 \mathrm{mg} / \mathrm{kg}$ thrice weekly i.p. or vehicle for 10 days (control plus vehicle, $n=6$; control plus GSK-J4, $n=6$; adriamycin plus vehicle, $n=6$; adriamycin plus GSK-J4, $n=6$ ). Values represent the mean \pm SEM. ${ }^{*} P<0.05,{ }^{* *} P<0.01$, ${ }^{* *} P<0.001$, and ${ }^{* * *} P<0.0001$, by 2-tailed Student's $t$ test (A-C and $\mathbf{C}$ ) and 1-way ANOVA followed by Fisher's LSD post-hoc test (D-F and I).

unaltered between the groups (Supplemental Figure 10C), and we were unable to detect evidence of apoptosis specifically within glomerular podocytes, either by transmission electron microscopy or by immunofluorescence microscopy to detect cleaved caspase-3 (Supplemental Figure 10D).
Podocyte H3K27me3 is diminished and UTX expression is increased in human glomerular disease. In our next series of experiments, we set out to determine whether H3K27me3, EZH2, Jmjd3, and/or UTX are altered in their distribution or expression in human glomerular disease. We examined 2 sets of archival kidney 
A

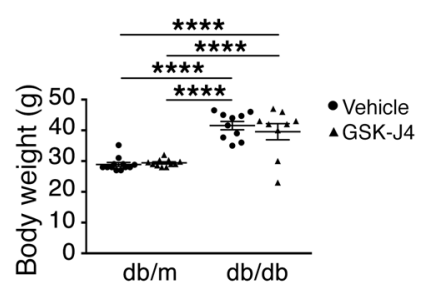

D

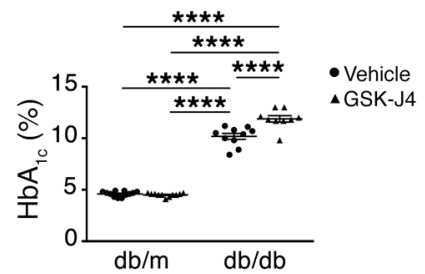

B

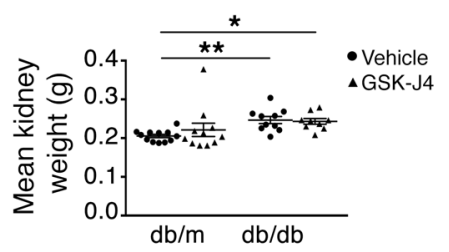

E

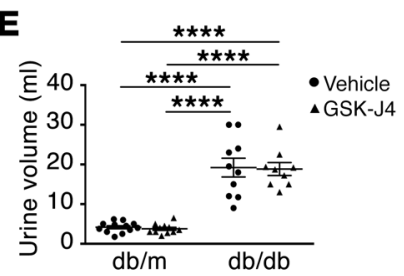

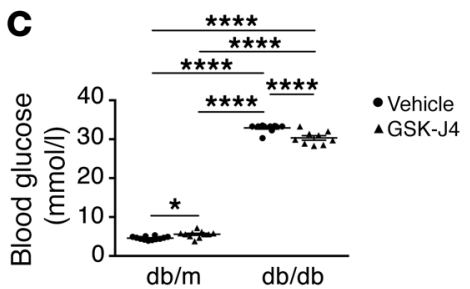

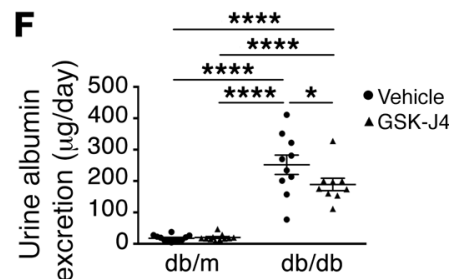

G
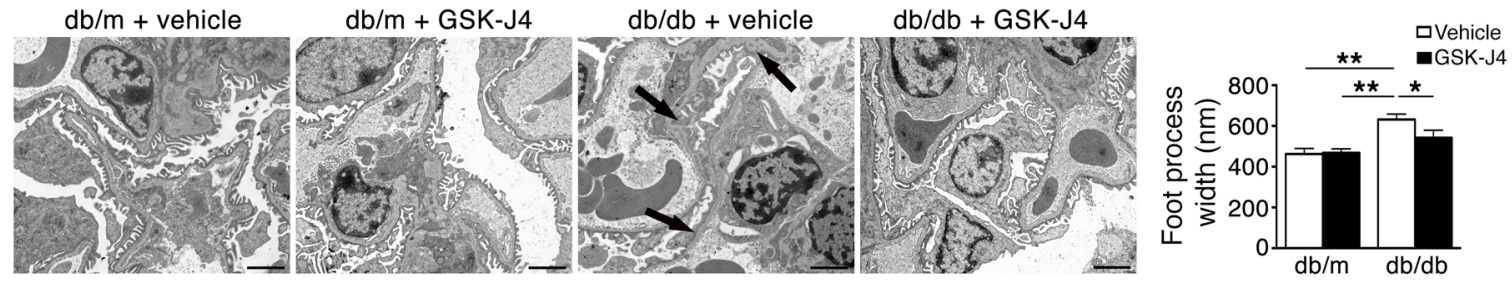

H
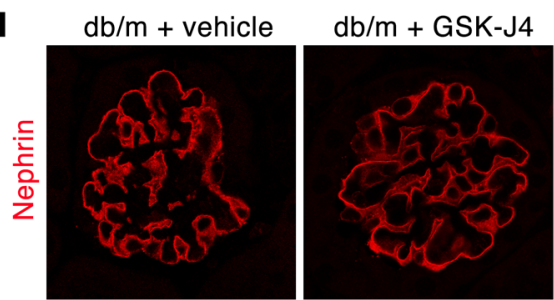

$\mathrm{db} / \mathrm{db}+$ vehicle
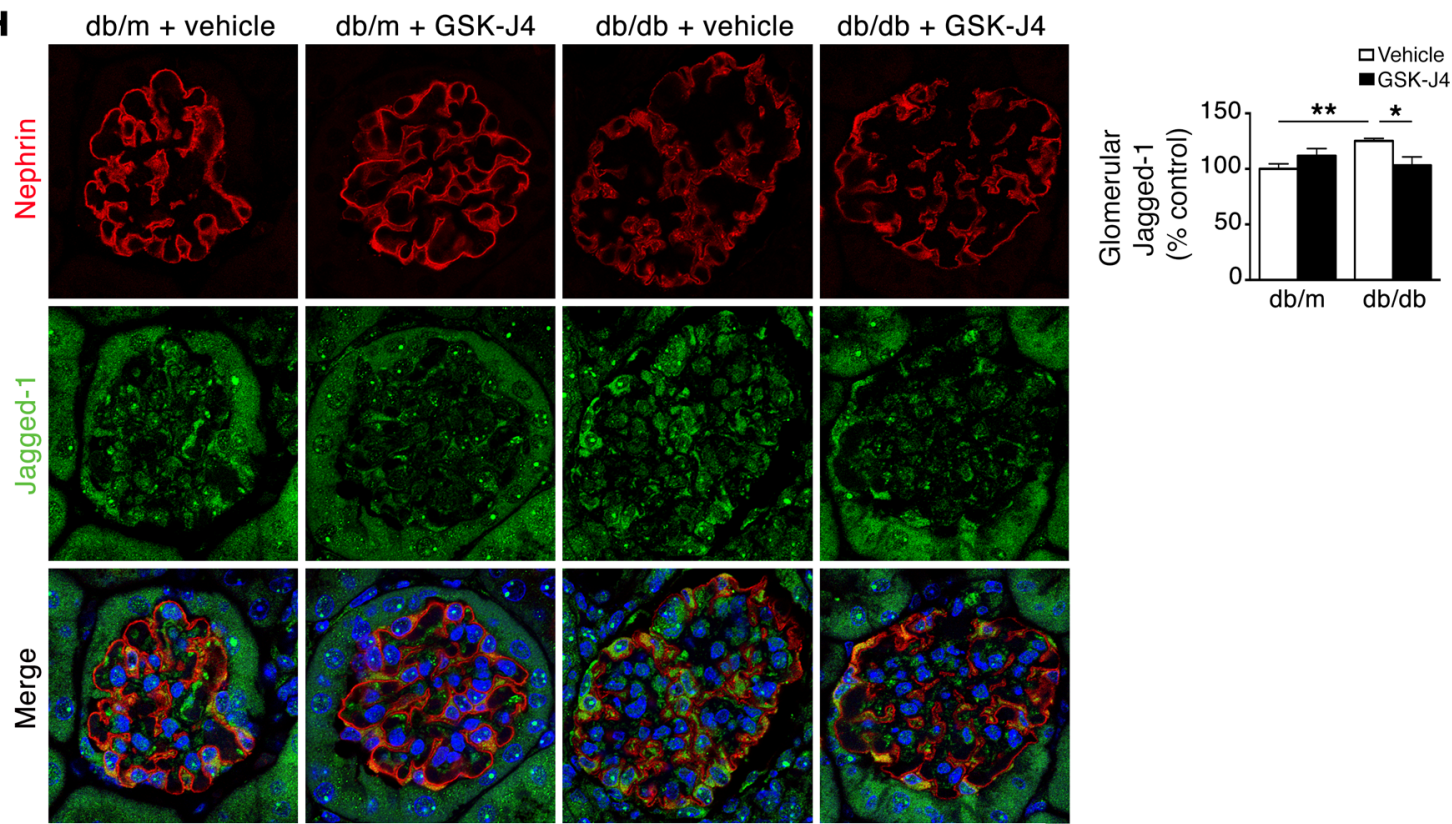

Figure 4. Inhibition of Jmjd3 and UTX attenuates renal injury in diabetic mice. (A) Body weight, (B) kidney weight, (C) blood glucose, (D) HbA ${ }_{1 c^{\prime}}(\mathrm{E})$ urine volume, and (F) 24-hour urine albumin excretion in nondiabetic $\mathrm{db} / \mathrm{m}$ mice and diabetic $\mathrm{db} / \mathrm{db}$ mice treated with vehicle or GSK-J4 (10 mg/kg) i.p. thrice weekly for 10 weeks ( $\mathrm{db} / \mathrm{m}$ plus vehicle, $n=12$; db/m plus GSK-J4, $n=11$; db/db plus vehicle, $n=10$; db/db plus GSK-J4, $n=9$ ). (G) Transmission electron micrographs and podocyte foot process widths for $\mathrm{db} / \mathrm{m}$ and $\mathrm{db} / \mathrm{db}$ mice treated with vehicle or GSK-J4 (db/m plus vehicle, $n=4$; $\mathrm{db} / \mathrm{m}$ plus $\mathrm{CSK}-J 4, n=4$; db/db plus vehicle, $n=4$; db/db plus GSK-J4, $n=4$ ). Arrows mark areas of foot process widening in the glomerulus of the vehicle-treated $\mathrm{db} / \mathrm{db}$ mouse. Scale bars: $2 \mu \mathrm{m}$. (H) Immunofluorescence to detect the podocyte marker nephrin and Jagged-1 in glomeruli from db/m and db/db mice treated with vehicle or GSK-J4 (db/m plus vehicle, $n=12$; db/m plus GSK-J4, $n=11 ; \mathrm{db} / \mathrm{db}$ plus vehicle, $n=9 ; \mathrm{db} / \mathrm{db}$ plus GSK-J4, $n=9$ ). DAPI staining is shown in blue. Original magnification, $\times 630$. Values represent the mean $\pm \mathrm{SEM}$. ${ }^{*} P<0.05,{ }^{* *} P<0.01$, and ${ }^{* * *} P<0.0001$, by 1 -way ANOVA followed by Fisher's LSD post-hoc test.

tissue: (a) tissue from individuals with histopathologically confirmed diabetic glomerulosclerosis, which was compared with tissue from individuals without diabetes or glomerular disease (each obtained at the time of tumor nephrectomy) (Supplemental Table
1); and (b) biopsy tissue obtained from patients with FSGS that was compared with implantation biopsy tissue taken at the time of living donor kidney transplantation (Supplemental Table 2 and Figure 5). Like in normal mice glomeruli, we found that many glomer- 
A

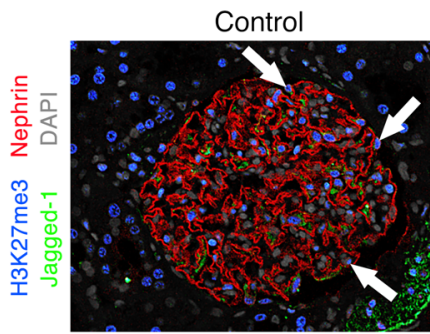

Diabetes

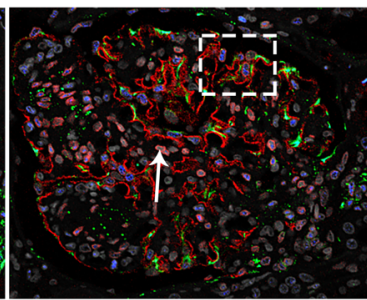

Zoom

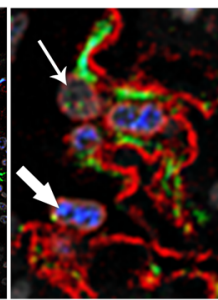

FSGS

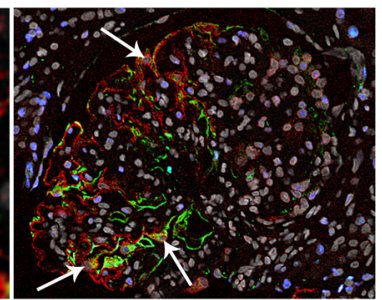

B

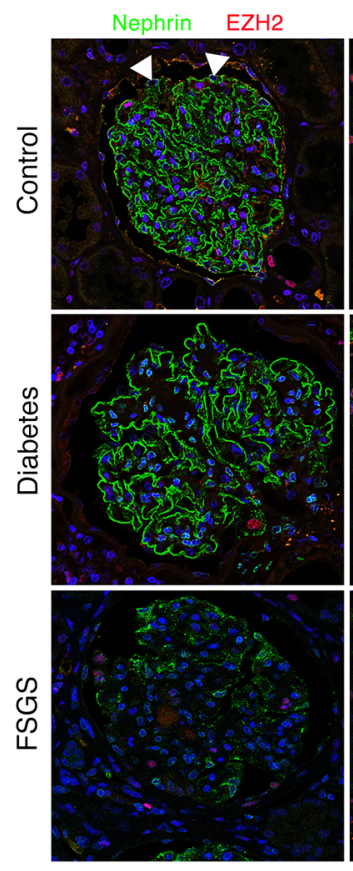

Nephrin Jmjd3

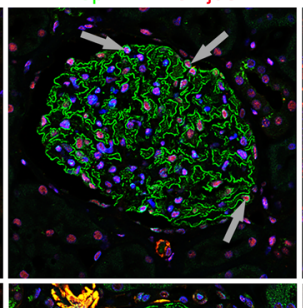

Nephrin

UTX

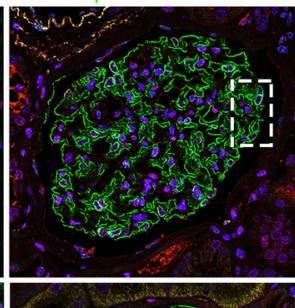

Zoom
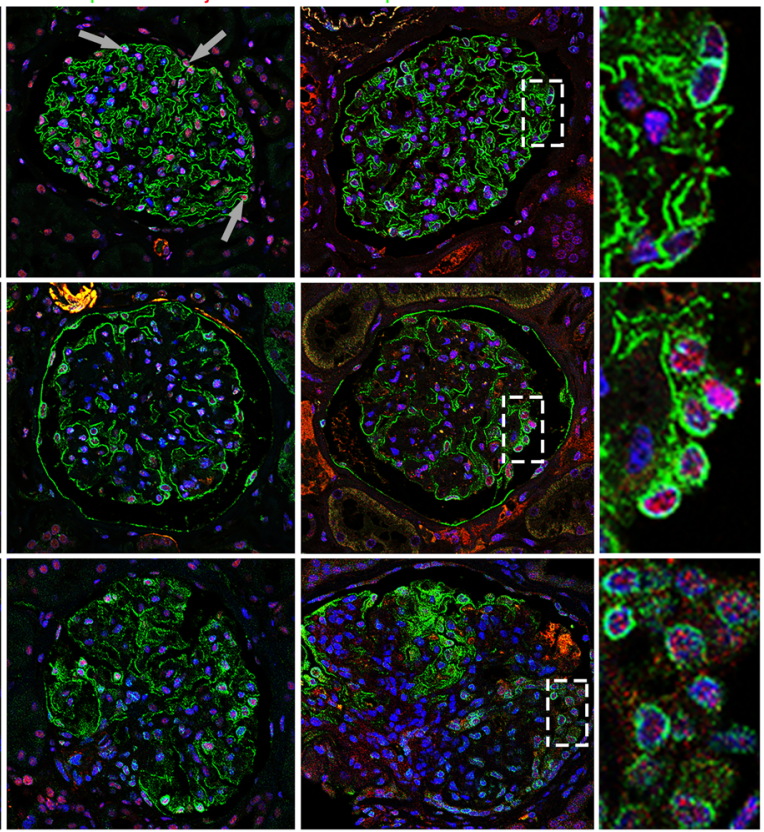

D

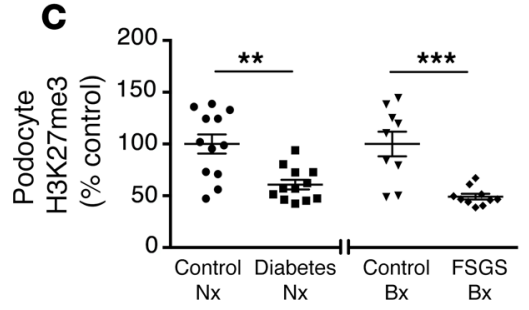

E

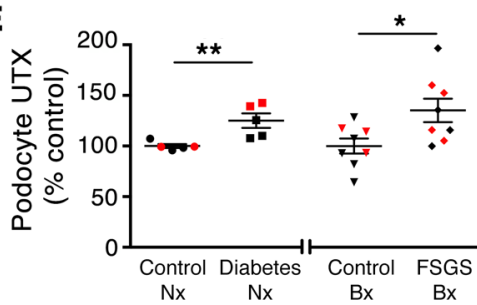

Figure 5. H3K27me3, Jagged-1, EZH2, Jmjd3, and UTX in normal human glomeruli and in glomerular disease. (A) Immunofluorescence to detect H3K27me3, the podocyte marker nephrin, and Jagged-1 in human kidney tissue (control nephrectomy, $n=12$; diabetic nephropathy nephrectomy, $n=12$; control biopsy, $n=9$; FSCS biopsy, $n=10$ ). In normal kidneys, H3K27me3 was present in the nuclei of numerous glomerular cells (and tubule epithelial cells), including nephrin-positive cells (podocytes, indicated by thick white arrows). In glomerular disease (diabetic nephropathy or FSCS), there was loss of H3K27me3, including from podocytes (thin white arrows), especially in more severely scarred glomeruli, that coincided with Jagged-1 expression and nephrin downregulation. The zoomed image is an enlargement of the outlined area. DAPI staining is shown in gray. (B) Immunofluorescence images for EZH2, Jmjd3 (control nephrectomy, $n=6$; diabetic nephropathy nephrectomy, $n=6$; control biopsy, $n=6$; FSGS biopsy, $n=6$ ) or UTX (control nephrectomy, $n=5$, diabetic nephropathy nephrectomy, $n=5$; control biopsy, $n=8$; FSCS biopsy, $n=8$ ) and nephrin in normal human glomeruli and in glomeruli from individuals with diabetic nephropathy or FSGS. Despite elevated podocyte H3K27me3 levels, EZH2 was absent from podocyte nuclei in control glomeruli (arrowheads) and glomeruli from patients with diabetic nephropathy or FSCS. In contrast, Jmjd3 was expressed by many glomerular cells, including podocytes (gray arrows), and its expression was unaltered in glomerular disease. UTX was also expressed in normal human podocytes, and its expression was significantly increased in podocytes from individuals with diabetic nephropathy or FSCS. The zoomed images are an enlargement of the outlined areas. DAPI staining is shown in blue. Original magnification, $\times 200$. (C-E) Quantification of podocyte H3K27me3 (C), Jmjd3 (D), and UTX (E) levels. Because UTX is considered to escape $X$ chromosome inactivation, the podocyte UTX levels in females are highlighted in red. Values represent the mean \pm SEM. ${ }^{*} P<0.05$, ${ }^{* *} P<0.01$, and ${ }^{* * *} P<0.01$, by 2 -tailed Student's $t$ test. Bx, biopsy; $N x$, nephrectomy.

ular nuclei immunostained positively for $\mathrm{H} 3 \mathrm{~K} 27 \mathrm{me} 3$ in normal human kidney sections (Figure 5A). In kidney sections from individuals with diabetic glomerulosclerosis, there was a progressive loss of podocyte H3K27me3 that coincided with glomerular inju- ry, Jagged-1 upregulation, and nephrin loss, whereas glomerular cell and tubule cell H3K27me3 content also appeared diminished (Figure 5, A and C). Likewise, there was an overall reduction in nuclear H3K27me3 levels in kidney sections from individuals with 


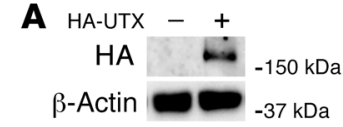

D

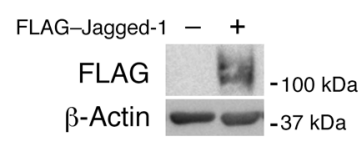

G

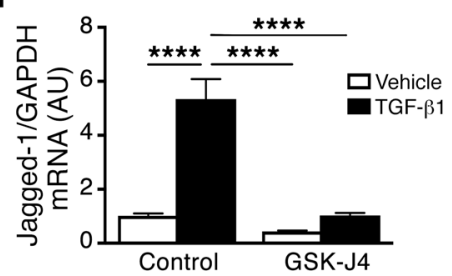

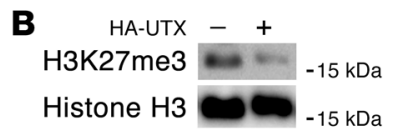
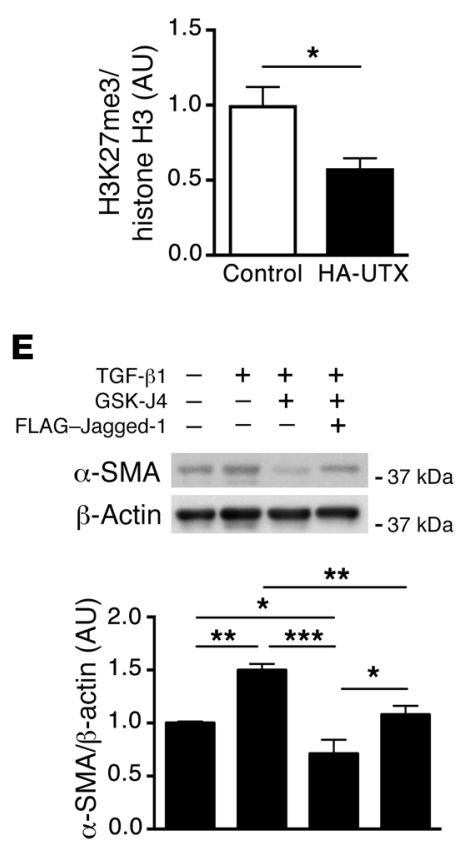
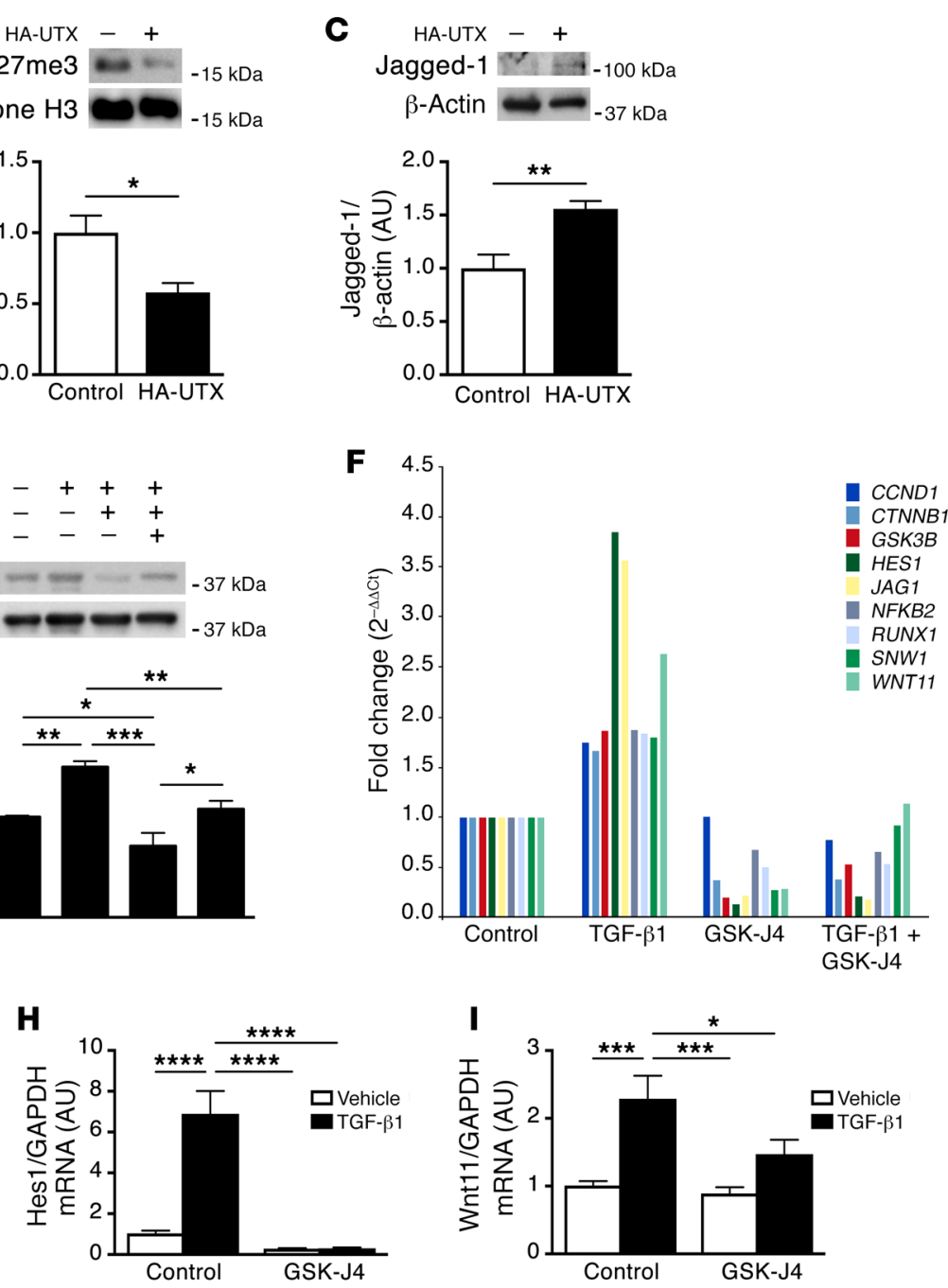

Figure 6. Shifts in H3K27me3 regulate Notch pathway activity in human podocytes. (A-C) Effect of UTX overexpression in differentiated immortalized human podocytes. (A) Transfection of human podocytes with HA-tagged human WT UTX. (B and C) Immunoblotting for H3K27me3 (B, $n=4$ ) and Jagged-1 $(\mathbf{C}, n=6)$ in UTX-overexpressing human podocytes. ( $\mathbf{D}$ and $\mathbf{E}$ ) Effect of Jagged-1 overexpression in differentiated human podocytes. (D) Transfection of human podocytes with FLAG-tagged human WT Jagged-1 $(n=3)$. (E) Effect of Jagged-1 overexpression on attenuating the actions of CSK-J4 ( $5 \mu$ M) in preventing TGF- $\beta 1$-induced ( $10 \mathrm{ng} / \mathrm{ml}$ for $48 \mathrm{~h}$ ) $\alpha$-SMA upregulation $(n=3)$. (F) Notch pathway-related genes upregulated by TCF- $\beta 1$ in human podocytes identified by RT ${ }^{2}$ Profiler PCR Array $(P<0.05$, Student's $t$ test) ( $n=4$ per condition). (G-I) mRNA levels of Jagged-1, Hes1, and Wnt11 in human podocytes treated with TCF- $\beta 1(10 \mathrm{ng} / \mathrm{ml})$ in the presence or absence of GSK-J4 $(5 \mu \mathrm{M})$ for 48 hours $(n=5)$. Values represent the mean \pm SEM. ${ }^{*} P<0.05,{ }^{* *} P<0.01$, ${ }^{* *} P<0.001$, and ${ }^{* * *} P<0.0001$, by 2-tailed Student's $t$ test (B and $\mathbf{C}$ ) and 1-way ANOVA followed by Fisher's LSD post-hoc test (E and G-I).

FSGS (Figure 5A), including a reduction in podocyte H3K27me3 levels (Figure 5C). Interestingly however, and unlike in mice glomeruli, despite abundant H3K27me3 levels in the podocytes of human glomeruli, EZH2 immunoreactivity was uncommon in human glomerular cells (Figure 5B). Despite the absence of EZH2 and the continued presence of H3K27me3, both Jmjd3 and UTX were expressed by human glomerular cells, including glomerular podocytes (Figure 5B). However, the expression patterns of the 2 demethylases varied with the presence of glomerular disease. Podocyte Jmjd3 immunoreactivity was abundant in control glomeruli and in glomeruli from patients with diabetic nephropathy or FSGS, with no difference in podocyte Jmjd3 expression between tissue phenotypes (Figure 5, B and D). In contrast, whereas low levels of UTX protein were detectable in podocyte nuclei from control tissue, podocyte nuclear UTX content was significantly increased in both diabetic glomeruli and the glomeruli of individuals with FSGS (Figure 5, B and E).

H3K27me3 regulates Jagged-1 expression and podocyte dedifferentiation in human podocytes. Having observed increased UTX expression, loss of H3K27me3, and increased Jagged-1 expression in the podocytes of individuals with glomerular disease, we queried whether UTX is causatively implicated in Jagged-1 upregulation. Accordingly, we transfected differentiated human podocytes (33) with a plasmid encoding HA-tagged human UTX (Figure 6A) and found that this led to a decrease in H3K27 trimethylation (Figure 6B) and an upregulation in Jagged-1 expression (Figure 6C), analogous to the human disease state. We then transfected human podocytes with FLAG-tagged human Jagged-1 (Figure 6D) and found that Jagged-1 overexpression partially blocked the actions of GSK-J4 in preventing TGF- $\beta 1-$ 


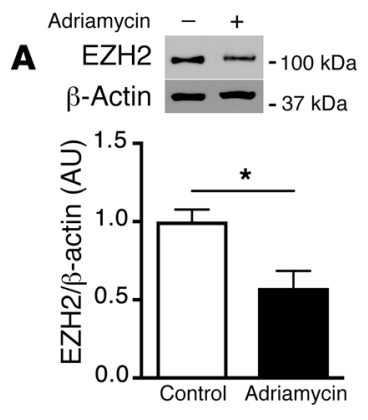

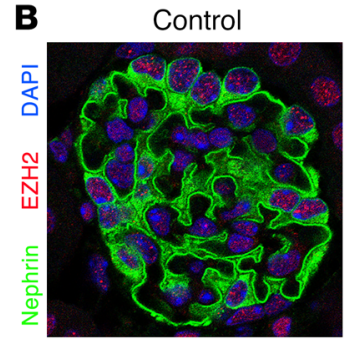
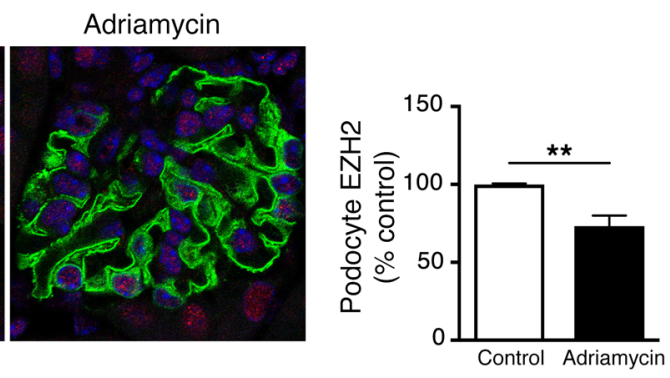

C
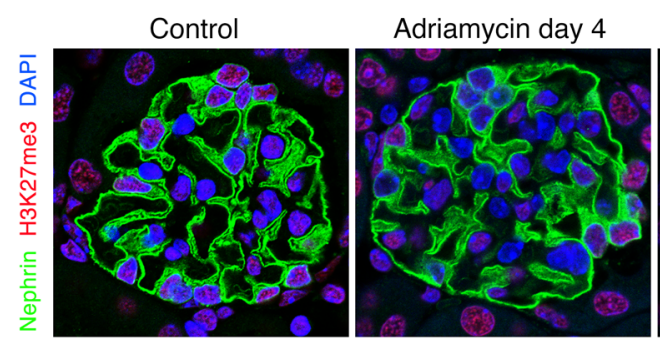

Adriamycin day 12

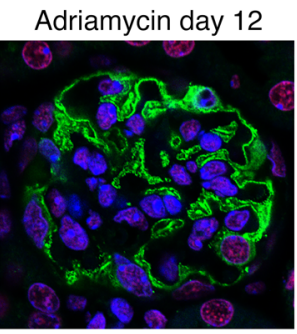

+ GSK-J4

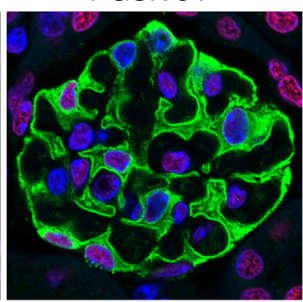

D

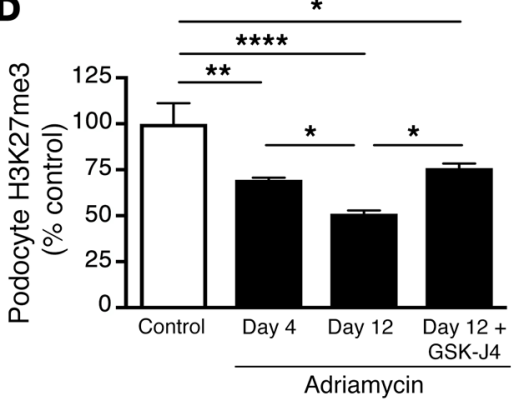

$\mathbf{E}$

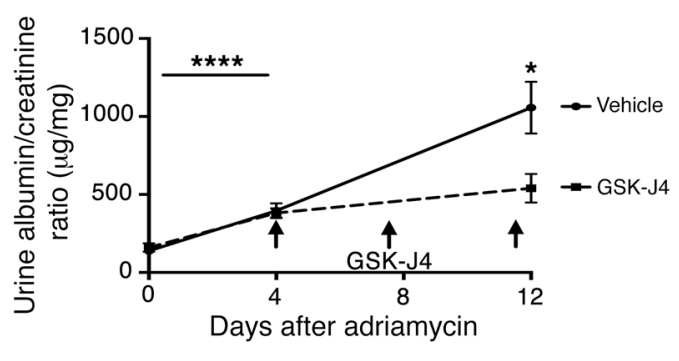

Figure 7. In mice with established glomerular disease, inhibition of Jmjd3 and UTX prevents both further podocyte H3K27me3 loss and albuminuria progression. (A) Immunoblotting for EZH2 in lysates of immortalized mouse podocytes after treatment with adriamycin $(300 \mathrm{ng} / \mathrm{ml}) \mathrm{for} 48 \mathrm{hours}(n=6)$. (B) Immunofluorescence for the podocyte protein nephrin and EZH2 in BALB/c mice 4 days after injection with vehicle $(n=4)$ or adriamycin $(n=4,10 \mathrm{mg}$ / $\mathrm{kg}$ ) and quantification of podocyte EZH2 levels. (C) Immunofluorescence staining for H3K27me3 and the podocyte marker nephrin in kidney sections from a normal control BALB/c mouse and from BALB/c mice 4 days and 12 days after adriamycin injection and from a BALB/c mouse 12 days after adriamycin injection and treatment with GSK-J4 (10 mg/kg i.p.) beginning on day 4. DAPI staining is shown in blue. Original magnification, $\times 630$. (D) Podocyte H3K27me3 content (control, $n=4$; adriamycin day 4, $n=4$; adriamycin day $12, n=4$; adriamycin plus GSK-J4, $n=4$ ). (E) Urine albumin/creatinine ratio in $\mathrm{BALB} / \mathrm{c}$ mice injected with adriamycin and treated with CSK-J4 $(10 \mathrm{mg} / \mathrm{kg})$ i.p. thrice weekly or with vehicle for 8 days, beginning 4 days after adriamycin injection (vehicle, $n=6$; GSK-J4, $n=7$ ). Arrows indicate GSK-J4 injections. Values represent the mean $\pm \mathrm{SEM} .{ }^{*} P<0.05,{ }^{* *} P<0.01$, and ${ }^{* * * *} P<0.0001$, by 2-tailed Student's $t$ test (A, B, and E) and 1-way ANOVA followed by Fisher's LSD post-hoc test (D).

induced $\alpha$-SMA upregulation (Figure 6E). Next, recognizing that Jagged-1 is unlikely to be the only Notch pathway member (or mediator of renal disease) that is regulated by H3K27me3 and recognizing that Jagged-1 overexpression attenuated but did not abrogate the effects of TGF- $\beta 1$, we probed for additional candidates using an $\mathrm{RT}^{2}$ Profiler PCR Array of 84 Notch pathwayrelated genes (Supplemental Table 3). We identified 9 Notch pathway-related genes (including JAG1) to be upregulated by TGF- $\beta 1$ in human podocytes, but not in human podocytes treated with GSK-J4 (CCND1, CTNNB1, GSK3B, HES1, JAG1, NFKB2, $R U N X 1, S N W 1$, and WNT11) (Figure 6F). We performed confirmatory reverse transcription quantitative PCR (RT-qPCR) of the 3 genes that were increased by more than 2-fold (JAG1 and the Notch target genes HES1 and WNT11), and in each case, GSK-J4 treatment negated the rise in $\mathrm{mRNA}$ levels induced by treatment with TGF- $\beta 1$ (Figure 6, G-I).
Inhibition of Jmjd 3 and UTX in mice with established glomerular disease analogous to human disease prevents nephropathy progression. Finally, we set out to determine what the effects of Jmjd3 and UTX inhibition would be in mice with preexisting glomerular injury and decreased H3K27me3 levels, analogous to the human disease setting. Incubation of cultured mouse podocytes with adriamycin caused a reduction of approximately $50 \%$ in podocyte EZH2 levels (Figure 7A), whereas podocyte EZH2 levels were also diminished in BALB/c mice 4 days after injection with adriamycin (Figure 7B). Correspondingly, injection of BALB/c mice with adriamycin caused a progressive loss of podocyte H3K27me3 content over 12 days (Figure 7, C and D) that coincided with the progressive development of albuminuria (Figure $7 \mathrm{E}$ ). We treated mice with GSK-J4, beginning 4 days after adriamycin injection, when podocyte EZH 2 and $\mathrm{H} 3 \mathrm{~K} 27 \mathrm{me} 3$ levels were already reduced and albuminuria was already established, thereby preventing both 

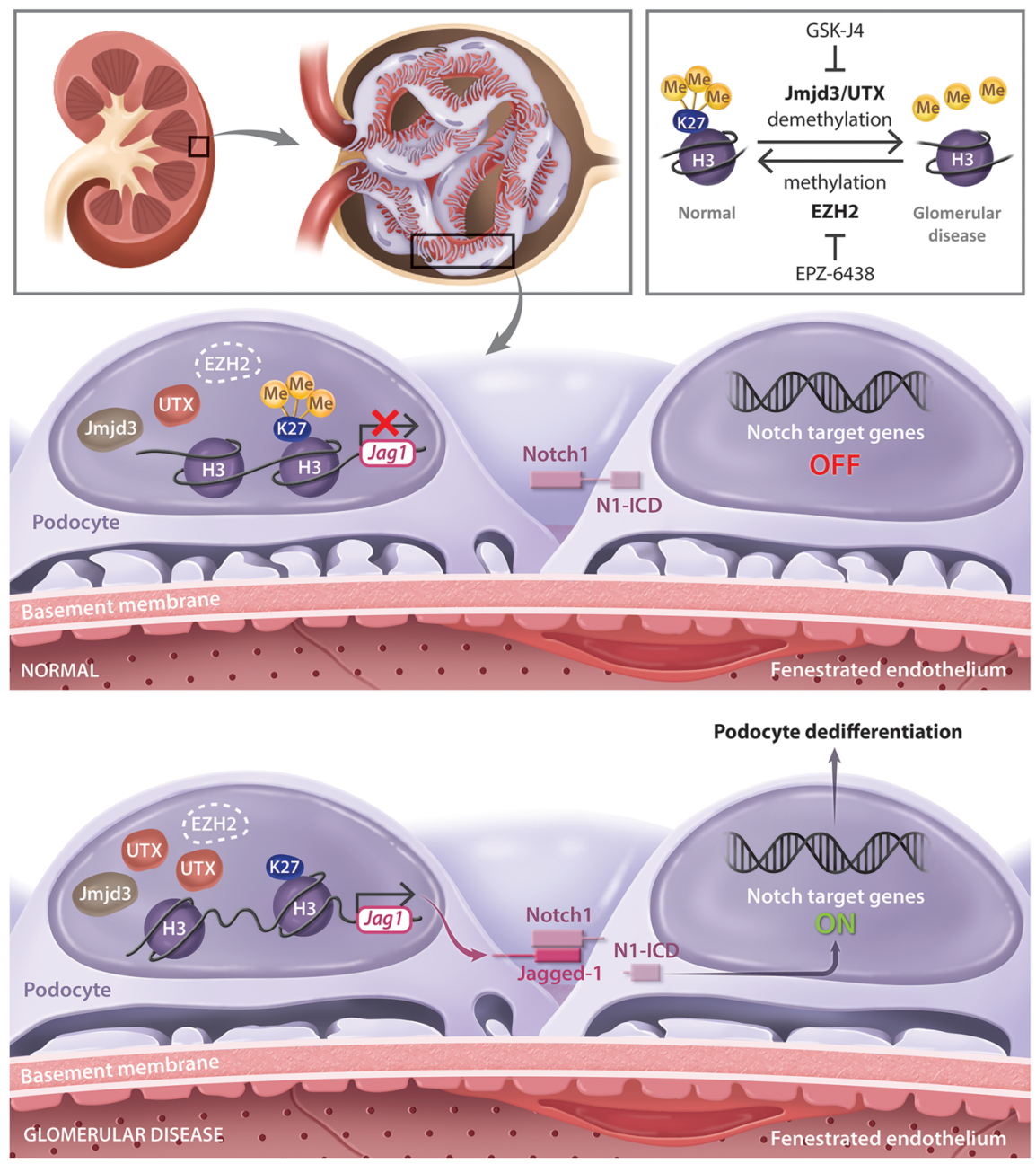

Figure 8. Mechanism by which histone patterning by H3K27me3 regulates podocyte differentiation or dedifferentiation in glomerular disease. The histone octamer is made up of 4 core histone proteins: $\mathrm{H} 2 \mathrm{~A}, \mathrm{H} 2 \mathrm{~B}, \mathrm{H3}$, and $\mathrm{H} 4$. Lysine residue 27 (K27) on histone $\mathrm{H} 3$ can be mono-, di-, or trimethylated. $\mathrm{H} 3 \mathrm{~K} 27$ trimethylation is catalyzed by the histone methyltransferase $\mathrm{EZH} 2$, and it is demethylated by the lysine-specific demethylases Jmjd3 and UTX. EPZ-6438 is a small-molecule inhibitor of EZH2 and thus decreases H3K27me3 levels. CSK-J4 is a small-molecule inhibitor of Jmjd 3 and UTX and increases H3K27me3 levels. In normal podocytes, $\mathrm{H} 3 \mathrm{~K} 27 \mathrm{me} 3$ is enriched at the promoter of the gene encoding the Notch ligand Jagged-1, repressing its expression and restraining Notch pathway activity. In human podocytes, this occurs even in the absence of EZH2 expression and in the presence of Jmjd3 and UTX. In glomerular disease, H3K27me3 is lost (associated with upregulation of UTX in human podocytes), and this results in derepression of Jagged-1. Expression of Jagged-1 at the cell surface then initiates juxtacrine signaling by binding to Notch1, which leads to cleavage of Notch1 at the membrane and nuclear translocation of the cleavage product N1-ICD. N1-ICD, together with its binding partners, promotes the expression of Notch target genes, resulting in podocyte dedifferentiation and subsequent glomerular disease. further podocyte H3K27me3 loss (Figure 7, C and D) and albuminuria progression (Figure $7 \mathrm{E}$ ). Similarly, we subjected mice to $\mathrm{SNx}$ surgery and, 6 weeks later, began treating the animals with GSK-J4 for an additional 4 weeks. Whereas glomerular Jagged-1 content and albuminuria were both increased in vehicle-treated SNx mice compared with sham-operated mice 10 weeks after surgery, these increases were abated with the delayed initiation of GSK-J4 treatment (Supplemental Figure 11).

\section{Discussion}

The strategies that the eukaryotic nucleus uses when regulating gene transcription extend well beyond the conventional cis/trans paradigm (34). Deposition of the repressive H3K27me3 mark during embryogenesis, for instance, limits the expression of genes that were once important for passage through a particular developmental stage but that are no longer required by the differentiated adult cell (5). Here, we discovered that loss of the H3K27me3 mark in quiescent adult glomerular podocytes initiates signaling through the developmentally critical Notch pathway and permits the occurrence of glomerular disease, whereas gain of H3K27me3 has the opposite effect. These actions are at least partly mediated by derepression or repression of the Notch ligand Jagged-1 (Figure 8). Given the increasing importance being placed on the reactivation of developmental pathways in the pathogenesis of fibrotic diseases (18), it would be prudent to also consider the epigenomic regulation of these pathways when exploring opportunities for therapeutic intervention.

The significance of chromatin modifications to the determination of the cellular phenotype is most easily appreciated during embryogenesis (35) or cancer development (36), which are times of rapid cell proliferation and differentiation or dedifferentiation. In contrast, the notion that these processes also influence the maintenance of the quiescent phenotype of differentiated cells has only recently emerged. In 2010, investigators observed that deleting a component of the histone $\mathrm{H} 3$ lysine 4 methyltransferase complex from podocytes resulted in progressive proteinuria (37). Since then, altered DNA methylation patterns (38) and posttranslational histone changes (38-40) in podocytes have each been shown to affect glomerular cell permselectivity. Here, we observed that either deletion of EZH2 specifically from podocytes or systemic inhibition of EZH2 activity diminished levels of the repressive H3K27me3 mark, provoked Notch pathway activation, and sensitized mice to glomerular disease. We found that H3K27me3 was enriched at the promoter region of the Notch ligand Jagged-1 and that the podocyte dedifferentiation that accompanied H3K27me3 diminution was Notch pathway dependent. The Notch pathway is a juxtacrine signaling cascade that is necessary for cell-fate determination during kidney development (28). It is largely dormant in the 
normal adult human glomerulus $(24,29)$. However, in the context of glomerular diseases, including FSGS and diabetic nephropathy, it can become reactivated, promoting podocyte dedifferentiation and accelerating glomerular damage $(24,25,29)$. During development, chromatin remodeling provides an important means by which a cell can set, coordinate, and integrate Notch-dependent processes (41). The findings of the present study indicate that chromatin remodeling events are similarly involved in the reactivation of developmental processes during the occurrence of glomerular disease. The mechanisms by which podocyte dedifferentiation causes glomerular disease remain a subject of debate. Whereas others have reported that Notch pathway reactivation is accompanied by podocyte apoptosis (24), here, we did not observe apoptosis specifically within podocytes at the time points measured. We cannot, however, exclude the relative contributions of podocyte detachment or podocyte dysfunction to the glomerular injury that accompanied podocyte dedifferentiation in the present study.

To explore the effects of increasing podocyte H3K27me3 content, we took advantage of a recently developed small-molecule inhibitor, GSK-J4, the cell-penetrating ethyl ester derivative of the Jmjd3 and UTX inhibitor GSK-J1 (30). The inhibitor acts by chelating the $\mathrm{Fe}$ (II) active site and by binding of a propanoic acid side-chain to the 2-oxoglutarate binding site of Jmjd3 and UTX (42). In vitro, we observed that GSK-J4 increased podocyte H3K27me3 content, repressed Jag1 expression, and prevented TGF- $\beta 1$-induced podocyte dedifferentiation. In vivo, we observed that GSK-J4 attenuated the development of glomerular disease induced by adriamycin, renal mass ablation, or diabetes and was accompanied by Jag1 repression and preservation of podocyte differentiation. In diabetic mice, we also detected subtle and variable shifts in dysglycemia with GSK-J4 treatment, including a marginal decrease in blood glucose but an increase in glycated hemoglobin levels. Although the metabolic effects of Jmjd3 and UTX are mostly unknown, UTX upregulation was recently shown to promote the browning of adipose tissue (43), and thus its inhibition could theoretically impair energy expenditure.

Consistent with a role for H3K27me3 loss in the development of glomerular disease, we observed a diminution in the histone mark in podocytes from mice as soon as 4 days after injection with adriamycin and in podocytes from humans with either diabetic nephropathy or FSGS. In humans with diabetic nephropathy, this coincided with upregulation in the expression of the demethylase UTX, whereas Jmjd3 levels were unaltered. This is interesting, because previous studies have suggested that Jmjd3 expression can be induced by stimuli such as hypoxia and inflammation, which are common features of CKD $(9,44)$. Moreover, although UTX is considered to escape X chromosome inactivation (45), we did not detect a difference in podocyte UTX levels between male and female patients. This observation is consistent with an earlier report that UTX expression from the inactive X chromosome is significantly lower than it is from the active $\mathrm{X}$ chromosome (46). Although UTX and Jmjd3 are similar in their JmjC catalytic domains, they are also structurally distinct. For instance, UTX possesses 6 protein interaction-mediating tetratricopeptide repeat domains, and Jmjd3 does not (13). Accordingly, the demethylases can have both overlapping and unique chromatin targets $(11,47)$, and they may also both have demethylase-independent roles in chromatin remodeling (48). However, the demethylating actions of UTX as a mediator of glomerular disease are collectively supported by the coincident loss of H3K27me3 in both human diabetic nephropathy and human FSGS and the attenuation of renal injury and podocyte dedifferentiation with GSK-J4 treatment in mice and cultured cells.

Unlike humans, mice are generally considered to be relatively resistant to the development of advanced glomerular disease. This differential sensitivity has been attributed to differences in the species-specific transcriptome (49). Here, the abundance of EZH2 protein in mouse podocytes in vivo and the absence of EZH2 from human podocytes in vivo indicate that differences also extend to the species-specific epigenome. Despite the absence of EZH2 and the persistence of Jmjd3 and UTX expression in adult human podocytes in vivo, and thus a catalytic nuclear environment that should favor demethylation, total podocyte nuclear H3K27me3 levels remained high. This finding is aligned with an earlier assertion that, in nonproliferating cells, persistent H3K27me3 expression could be a consequence of its inherent stability, its protection from demethylases by PRC1 and PRC2-EZH1-mediated chromatin compaction, and by the low-level H3K27 trimethylating activity of PRC2-EZH1 (50). In human glomerular disease, however, the epigenetic landscape appears to shift. This manifests as a progressive loss of $\mathrm{H} 3 \mathrm{~K} 27 \mathrm{me} 3$ and a sustained upregulation of UTX. It is unclear whether H3K27me3 loss in humans occurs as a consequence of chromatin-remodeling events that enable constitutively expressed Jmjd3 and UTX to erase previously concealed H3K27me3 mark(s) or whether coincident upregulation of UTX is a prerequisite. At least in cultured human podocytes, however, overexpression of UTX, without any external stimulus to precipitate chromatin remodeling, was sufficient to cause both loss of H3K27me3 and derepression of the H3K27me3-regulated Notch ligand JAG1. Correspondingly, in mice with established glomerular injury and diminished podocyte $\mathrm{H} 3 \mathrm{~K} 27 \mathrm{me} 3$ content, analogous to the human disease setting, Jmjd3 and UTX inhibition abated further H3K27me3 loss and prevented nephropathy progression.

Although we focused on the epigenetic regulation of podocyte Jagged-1, we recognized that JAG1 was unlikely to be the sole gene involved in podocyte dedifferentiation that is affected by H3K27me3 loss. In a leukemic cell line, for example, GSK-J4 treatment was accompanied by downregulation in 486 transcripts, among them Notch1 (47). In other cell types, H3K27me3 may have different roles in regulating Notch pathway activity. For instance, in adult cardiomyocytes, H3K27me3 can be found at the promoter regions of several silenced genes belonging to the Notch pathway, including Hes1, Hey1, and Notch1 itself (51). In contrast, in effector T cells, EZH2 aids Notch pathway activation by repressing the expression of the Notch repressors Numb and Fbxw7 (52). In a focused search for other known mediators of glomerular disease that may be influenced by shifts in podocyte H3K27me3 levels, we performed a PCR array for Notch pathway-related genes in human podocytes. This array not only identified both JAG1 and the Notch target gene HES1 as being upregulated by TGF- $\beta 1$ in a manner that was negated by Jmjd 3 and UTX inhibition, but it also identified the Wnt pathway member WNT11. Like Notch, the Wnt pathway is a developmentally essential signaling cascade that has been implicated in podocyte dedifferentiation (53). Wnt11 is a noncanonical 
Wnt protein that is known to crosstalk with the Notch signaling pathway (54) and that is expressed in the kidney at the tips of the ureteric buds during development (55). Its activation by TGF- $\beta$ promotes mesenchymal gene expression by tubule epithelial cells (56), although the specific actions of Wnt11 in adult podocytes are largely yet to be defined. The repression of WNT11 by Jmjd3 and UTX inhibition, directly or indirectly, raises the prospect that shifts in H3K27me3 have broader effects on developmental pathway reactivation that extend beyond those of the Notch pathway alone. Nonetheless, the partial reversal of the effects of inhibition of both Jmjd3 and UTX by Jagged-1 overexpression in vitro indicates that the association between $\mathrm{H} 3 \mathrm{~K} 27 \mathrm{me} 3$ loss and podocyte dedifferentiation, in the present study, is at least partially mediated by derepression of Jagged-1.

In summary, the H3K27me3 mark can be dynamically regulated either pharmacologically or by disease processes in adult differentiated, quiescent cells. In the adult glomerulus, loss of $\mathrm{H} 3 \mathrm{~K} 27 \mathrm{me} 3$ promotes the dedifferentiation of postmitotic podocytes and accelerates glomerular disease, whereas gain of H3K27me3 has the opposite effect. These actions involve the derepression or repression of the Notch ligand Jagged-1. Changes in histone patterning play a fundamental role in the development of glomerular disease, and inhibition of lysine-specific demethylases may favorably improve histone patterning during the progression of glomerular disease.

\section{Methods}

\section{Animal studies}

EZH2 $2^{\text {podKo }}$ mice. Podocin-Cre $e^{+}$mice [B6.Cg-Tg(NPHS2-cre)295Lbh/J] (19) were obtained from The Jackson Laboratory. Restriction of Cre recombinase expression to the renal glomerulus was confirmed by breeding podocin- $C r e^{+}$mice with $R 26 R^{f l / f l}$ reporter mice [B6;129S4-Gt(ROSA)26Sor ${ }^{\text {tm1sor } / J ; ~ T h e ~ J a c k s o n ~ L a b o r a t o r y] ~(57) . ~}$ $E z h 2^{f / f l}$ mice (B6;129P2-Ezh2 $2^{\text {tmlTara }} / \mathrm{Mmnc}$ ) (20) were generated by Alexander Tarakhovsky (The Rockefeller University, New York, New York, USA) and were obtained from the Mutant Mouse Resource and Research Center (MMRRC) at the University of North Carolina (Chapel Hill, North Carolina, USA).

Adriamycin toxicity. EZH2 $2^{\mathrm{Ctrl}}$ and $\mathrm{EZH} 2^{\mathrm{podKo}}$ mice, aged 8 to 10 weeks, received a single tail-vein injection of adriamycin $(25 \mathrm{mg} /$ $\mathrm{kg}$; MedChemExpress) (58) in PBS or PBS alone (vehicle) and were followed for 10 days. In separate studies, after receiving a tail-vein injection of adriamycin (or vehicle), $\mathrm{EZH} 2^{\mathrm{Ctrl}}$ and $\mathrm{EZH} 2^{\text {podKo }}$ mice were further randomly allocated to receive either DAPT $(20 \mathrm{mg} / \mathrm{kg}$; MedChemExpress) (59) in corn oil, $5 \% \mathrm{v} / \mathrm{v}$ ethanol, or vehicle alone by i.p. injection every 3 days (total of 3 injections). Eight-week-old male $\mathrm{BALB} / \mathrm{c}$ mice (Charles River Laboratories) received a single tail-vein injection of adriamycin (10 mg/kg) (60) in PBS or of PBS alone and were then further randomized to receive either EPZ-6438 (MedChemExpress) $(100 \mathrm{mg} / \mathrm{kg}$ [ref. 61] in $0.5 \%$ sodium carboxymethylcellulose and $0.1 \%$ Tween-80) or vehicle alone by once-daily oral gavage for 10 days. Alternatively, male BALB/c mice received a tail-vein injection of adriamycin $(10 \mathrm{mg} / \mathrm{kg})$ or PBS and were then randomly allocated to receive a thrice-weekly i.p. injection of GSK-J4 $(10 \mathrm{mg} / \mathrm{kg}$; MedChemExpress) in $0.1 \%$ DMSO in PBS or of 0.1\% DMSO in PBS alone for 10 days (total of 5 injections). In-house experiments revealed that this dos- ing regimen for GSK-J4 was well tolerated by mice and accompanied by a significant increase in renal H3K27me3 levels (Figure 3G). In the late-intervention study, male $\mathrm{BALB} / \mathrm{c}$ mice received a tail-vein injection of adriamycin $(10 \mathrm{mg} / \mathrm{kg})$ or PBS as described above. Four days later, mice were stratified by albuminuria level to receive GSK-J4 (10 mg/ $\mathrm{kg}$ ) or vehicle by i.p. injection on days 4, 7, and 11 (total of 3 injections).

SNX studies. Eight-week-old EZH $2^{\text {Ctrl }}$ and EZH $2^{\text {podKo }}$ mice underwent SNx (or sham surgery) using a surgical technique we have previously applied in rats (62). Briefly, under isoflurane anesthesia, the right kidney was removed by subcapsular nephrectomy, and 2 of the 3 or 4 branches of the left renal artery were selectively ligated. The sham surgery entailed laparotomy, manipulation of both kidneys, and wound closure. The sham-and SNx-operated EZH2 ${ }^{\mathrm{Ctrl}}$ and EZH2 ${ }^{\text {podko }}$ mice were followed for 6 weeks. In separate studies, 8-week-old male C57BL/6 mice (The Jackson Laboratory) underwent sham or SNx surgery, as described, and were followed for 6 weeks before randomization to receive either GSK-J4 $(10 \mathrm{mg} / \mathrm{kg}$ by thrice-weekly i.p. injections) in 0.1\% DMSO in PBS or 0.1\% DMSO in PBS alone for an additional 4 weeks.

Diabetic mouse studies. Eight-week-old male $\mathrm{db} / \mathrm{m}$ and $\mathrm{db} / \mathrm{db}$ mice on a BKS background (The Jackson Laboratory) were randomly allocated to receive either GSK-J4 (10 mg/kg by thrice-weekly i.p. injections) in $0.1 \%$ DMSO in PBS or 0.1\% DMSO in PBS alone and were followed for 10 weeks.

Urine albumin excretion was determined by ELISA (Assaypro) for the EZH2 $2^{\text {podKo }}$ and BALB/c mouse studies and Albuwell M ELISA (Exocell) for the $\mathrm{db} / \mathrm{db}$ mouse study after individually housing the mice in metabolic cages for 24 hours. Urine creatinine was determined with a Creatinine Companion Kit (Exocell). Blood glucose levels were determined using a OneTouch UltraMini meter (LifeScan Canada Ltd.; Burnaby, British Columbia, Canada). Hemoglobin A1c $\left(\mathrm{HbA}_{1 \mathrm{c}}\right)$ was determined using A1CNow Plus (Roxon Meditech Ltd.).

\section{Human tissue studies}

Archival formalin-fixed, paraffin-embedded kidney tissue from 12 patients with diabetic nephropathy and 12 individuals without diabetes, matched for age and sex, was examined. The tissue had been obtained at the time of nephrectomy for conventional renal carcinoma, with kidney tissue examined from regions of the kidney unaffected by tumor. Archival formalin-fixed, paraffin-embedded kidney tissue from 10 patients with FSGS obtained at the time of diagnostic biopsy was also examined and compared with archival tissue from 9 living kidney donors that was obtained before implantation. None of the patients with FSGS had concomitant diabetes mellitus. Sufficient tissue was available to enable the study of H3K27me3 levels in each kidney section, Jmjd3 levels in 6 kidney sections per group, and UTX levels in 5 kidney sections per group for the diabetic nephropathy study and in 8 kidney sections per group for the FSGS study. EZH2 immunostaining was assessed in a total of 11 control samples, 6 kidney sections from individuals with diabetic nephropathy, and 5 kidney sections from individuals with FSGS.

\section{Tissue immunofluorescence microscopy}

Mouse kidney sections. Immunofluorescence microscopy was performed on formalin-fixed, paraffin-embedded mouse kidney sections with the following antibodies: H3K27me3 rabbit monoclonal antibody, 1:200 (9733S; Cell Signaling Technology); EZH2 rabbit mono- 
clonal antibody, 1:100 (5246S; Cell Signaling Technology); N1-ICD rabbit polyclonal antibody, 1:100 (ab8925; Abcam); Jagged-1 rabbit polyclonal antibody, 1:100 (ab7771; Abcam); Alexa Fluor 488 donkey anti-rabbit secondary antibody, 1:100 (A21206, Thermo Fisher Scientific); nephrin goat polyclonal antibody, 1:100 (AF3159; R\&D Systems); Alexa Fluor 555 donkey anti-goat secondary antibody, 1:200 (ab150134, Abcam); and cleaved caspase-3 rabbit polyclonal antibody, 1:100 (9661S; Cell Signaling Technology). Immunofluorescence microscopy for WT1 rabbit polyclonal antibody, 1:200 (sc-192; Santa Cruz Biotechnology) and N1-ICD or WT1 and Hey1 rabbit polyclonal antibody, 1:75 (ab22614; Abcam) was performed using the following secondary antibodies: Alexa Fluor 488 donkey anti-rabbit antibody, 1:100 (Thermo Fisher Scientific) for WT1 and Alexa Fluor 647 Fab Fragment goat anti-rabbit antibody, 1:200 (111-607-003, Jackson ImmunoResearch) for N1-ICD or Hey1. DAPI (Cell Signaling Technology) was used at a concentration of 1:10,000.

Human kidney sections. Immunofluorescence microscopy was performed on archival formalin-fixed, paraffin-embedded kidney tissue sections with the following antibodies: H3K27me3, 1:200 (Cell Signaling Technology); EZH2, 1:75 (Cell Signaling Technology); Jmjd3 rabbit polyclonal antibody, 1:100 (ab38113; Abcam); UTX rabbit monoclonal antibody, 1:50 (33510S; Cell Signaling Technology); Alexa Fluor 555 donkey anti-rabbit secondary antibody, 1:100 (A31572, Thermo Fisher Scientific); Jagged-1, 1:100 (Abcam); Alexa Fluor 488 donkey antirabbit secondary antibody, 1:100 (Thermo Fisher Scientific); nephrin mouse monoclonal antibody, 1:100 (377246; Santa Cruz Biotechnology); and Alexa Fluor 647 donkey anti-mouse secondary antibody, 1:100 (A31571, Thermo Fisher Scientific). DAPI was used at a concentration of 1:10,000.

Slides were visualized on a Zeiss LSM 700 confocal microscope (Carl Zeiss Canada). All mice glomeruli were studied at $\times 630$ original magnification, and all human glomeruli were studied at $\times 200$ original magnification. For Jagged-1 and nephrin analysis in mouse tissue, the mean fluorescence intensity (MFI) was determined in 6 glomeruli from 5 to 6 mice per group using Fiji (63) and is represented as the percentage of change relative to the control. For determination of podocyte nuclear H3K27me3, UTX, and Jmjd3 levels in kidney tissue, podocyte nuclei were recognized by nephrin expression within the cell body (red for H3K27me3 analysis in humans; green for Jmjd3 and UTX analysis in humans and EZH2 analysis in mice). The cell nucleus was outlined using the free-hand tool in ImageJ software (NIH) (64), and the MFI was determined in approximately 10 podocytes per glomerulus in humans (4-14 podocytes per glomerulus in mice) and in an average of 6 glomeruli per case. All histological analyses were performed by investigators blinded to the study groups. Data are represented as the percentage of change relative to the control.

\section{Primary culture of podocytes}

For primary podocyte cultures, mouse glomeruli were first isolated with Dynabeads (Thermo Fisher Scientific). Under isoflurane anesthesia, the abdominal aorta was cannulated with a 24-gauge angiocath, and the mouse was perfused with $1 \times 10^{5}$ Dynabeads in $5 \mathrm{ml} \mathrm{HBSS}$ (Thermo Fisher Scientific). Podocytes were isolated using previously reported methods $(57,65,66)$. In brief, glomeruli were seeded on collagen I-coated plates in a 1:1 mixture of F-12 Kaighn's Modification Media (HyClone Laboratories) with media harvested from NIH/3T3 cells (ATCC), and cell cultures were maintained for approximately 4 to 6 days.

\section{Immunoblotting}

Immunoblotting was performed on cultured cell extracts with the following antibodies: EZH2, 1:1,000 (Cell Signaling Technology); $\beta$-actin mouse monoclonal antibody, 1:10,000 (A1978; Sigma-Aldrich); H3K27me3, 1:1,000 (Cell Signaling Technology); histone H3 rabbit polyclonal antibody, 1:2,000 (9715S; Cell Signaling Technology); N1-ICD, 1:1,000 (Abcam); Notch1 cleaved transmembrane/intracellular region (NTM) rabbit monoclonal antibody, 1:1,000 (3608S; Cell Signaling Technology); Jagged-1, 1:1,000 (Abcam); FLAG rabbit polyclonal antibody, 1:1,000 (2368S; Cell Signaling Technology); $\alpha$-SMA rabbit polyclonal antibody, 1:2,000 (ab5694; Abcam); Jmjd3, 1:1,000 (Abcam); UTX, 1:1,000 (Cell Signaling Technology); HA mouse monoclonal antibody, 1:500 (H3663; Sigma-Aldrich); H3K4me1 rabbit polyclonal antibody, 1:1,000 (ab8895; Abcam); and H3K4me3 rabbit polyclonal antibody, 1:1,000 (NB21-1023; Novus Biologicals). Densitometric analysis was performed using ImageJ software.

\section{Transmission electron microscopy}

Transmission electron microscopy was performed on kidney cortical tissue (Philips CM100; Electron Microscopy Research Services, Newcastle University). Images of the entire glomerular profile were taken through 3 randomly selected glomeruli from 4 mice per group. Foot process width was measured in a masked manner in approximately 10 micrographs (original magnification, $\times 5,800$ ) from each glomerular profile using ImageJ software. Podocyte density was determined using the method of Weibel and Gomez (67) in glomeruli from 4 mice per group. Evidence of apoptosis (crescentic pooling of nuclear chromatin) was sought in an average of 10 podocyte nuclei per glomerulus and in 3 glomeruli from 4 mice per group.

\section{Mesangial matrix index}

The magnitude of mesangial matrix accumulation was estimated using a semiquantitative scoring system on approximately 50 glomeruli in periodic acid-Schiff-stained (PAS-stained) kidney sections from 3 to 8 mice per group for adriamycin- and vehicle-treated mice and 9 to 12 mice per group for $\mathrm{db} / \mathrm{db}$ and $\mathrm{db} / \mathrm{m}$ mice, as previously described and by investigators blinded to the study groups (68).

\section{IHC}

IHC for $\alpha$-SMA (1:200; Abcam) was performed on 4- $\mu$ m-thick formalinfixed, paraffin-embedded kidney sections $(n=3-9 /$ group for adriamycin- and vehicle-treated mice and $n=9-12 /$ group for $\mathrm{db} / \mathrm{db}$ and $\mathrm{db} / \mathrm{m}$ mice). Glomerular immunostaining was quantified in a masked manner using ImageScope 11.1 software (Leica Microsystems) in approximately 30 glomeruli per kidney section. Data are expressed as the percentage change compared with the control. IHC for WT1 (1:900; Santa Cruz Biotechnology) was performed on 4 - $\mu$ m-thick formalin-fixed, paraffin-embedded kidney sections ( $n=4-9 /$ group for adriamycin- and vehicle-treated mice and $n=9-12 /$ group for db/ $\mathrm{db}$ and $\mathrm{db} / \mathrm{m}$ mice). Glomerular WT1 was determined by counting positively immunostained nuclei in 30 glomerular profiles (at the hilar level) in each kidney section. TUNEL staining was performed by the members of the Pathology Research Program at Toronto General Hospital (Toronto, Ontario, Canada). Glomerular TUNEL-positive nuclei were counted in an average of approximately 100 glomeruli per kidney section in 3 to 7 mice per group for adriamycin- and vehicletreated mice and 9 to 12 mice per group for $\mathrm{db} / \mathrm{m}$ and $\mathrm{db} / \mathrm{db}$ mice. 


\section{Cell culture}

Conditionally immortalized mouse podocytes. Differentiated, conditionally immortalized mouse podocytes were cultured as previously described (69). Cells were serum starved for 4 hours, followed by treatment with EPZ-6438 $(10 \mu \mathrm{M})(70)$ or GSK-J4 $(5 \mu \mathrm{M})(71)$ for 48 hours. To determine the effect of $\gamma$ secretase inhibition on EPZ-6438-induced podocyte dedifferentiation, cells were serum starved for 4 hours before coincubation with DAPT $(10 \mu \mathrm{M})(72)$ and EPZ-6438 $(10 \mu \mathrm{M})$ for 72 hours. For Notch1 knockdown, cells were transfected with $50 \mathrm{nM}$ mouse sequencespecific siRNA (Thermo Fisher Scientific) for 24 hours and then treated with EPZ-6438 $(10 \mu \mathrm{M})$ for a further 72 hours. In separate experiments, after 4 hours of serum starvation, cells were treated with GSK-J4 $(5 \mu \mathrm{M})$ for 30 minutes before the addition of recombinant human TGF- $\beta 1$ (10 ng/ml; Bio-Rad) (31) for 48 hours. For knockdown of EZH2, cells were transfected with $1 \mu \mathrm{g} / \mathrm{ml}$ mouse EZH2 sequence-specific shRNA plasmid (QIAGEN) or empty vector plasmid for 48 hours. For knockdown of Jmjd 3 and UTX, cells were transfected with $50 \mathrm{nM}$ mouse sequencespecific siRNA (Santa Cruz Biotechnology) or scrambled siRNA (Thermo Fisher Scientific) for 48 hours. For adriamycin treatment, cells were incubated with $300 \mathrm{ng} / \mathrm{ml}$ adriamycin for 48 hours. For N1-ICD immunofluorescence staining, cells were stained with N1-ICD antibody, 1:100 (Abcam); Alexa Fluor 555 donkey anti-rabbit secondary antibody, 1:100 (Thermo Fisher Scientific); and DAPI (1:10,000).

Conditionally immortalized human podocytes. Immortalized human podocytes were provided by Moin A. Saleem (University of Bristol, Bristol, United Kingdom) and cultured and differentiated as previously described (33). Human WT pCMV-HA-UTX was a gift of Kristian Helin (Biotech Research and Innovation Centre, University of Copenhagen, Copenhagen, Denmark) (plasmid 24168; Addgene). For UTX overexpression, cells were transfected with $500 \mathrm{ng} / \mathrm{ml} \mathrm{pCMV-HA-UTX} \mathrm{for} 48$ hours. For Jagged-1 overexpression, cells were transfected overnight with $500 \mathrm{ng} / \mathrm{ml}$ human WT Jagged-1 inserted into a pCMV3-C-FLAG vector (Sino Biological). Cells were then incubated with GSK-J4 $(5 \mu \mathrm{M})$ for 30 minutes before the addition of recombinant human TGF- $\beta 1$ (10 $\mathrm{ng} / \mathrm{ml}$ ) for an additional 48 hours. The $\mathrm{RT}^{2}$ Profiler PCR Array (PAHS059Z; QIAGEN) was used to identify Notch pathway-related genes that were altered in human podocytes by TGF- $\beta 1$ (10 ng/ml for 48 hours) and affected by GSK-J4 ( $5 \mu \mathrm{M}$, administered 30 minutes before the addition of TGF- $\beta 1$ ). Analysis of transcriptome profiles was performed using RT2 ${ }^{2}$ Profiler PCR Array Data Analysis, version 3.5 (QIAGEN). Values were normalized to the geometric mean of GAPDH and RPLPO.

\section{RT-qPCR}

RNA isolation from cell extracts was performed using TRIzol Reagent (Life Technologies, Thermo Fisher Scientific). cDNA was reverse transcribed from $2 \mu \mathrm{g}$ total RNA using a cDNA High Capacity Reverse Transcription Kit (Thermo Fisher Scientific). Primer sequences (Supplemental Table 4) were designed and validated using Primer-BLAST (http://www.ncbi.nlm.nih.gov/tools/primer-blast/) and synthesized by Integrated DNA Technologies. RT-qPCR was performed using SYBR Green on a ViiA7 Real-Time PCR System (Thermo Fisher Scientific). Experiments were performed at least in triplicate, and data analyses were performed using the Applied Biosystems Comparative $\mathrm{C}_{\mathrm{T}}$ Method.

\section{ChIP}

The ChIP assay was performed using an EZ-Magna ChIP A Chromatin Immunoprecipitation Kit (MilliporeSigma). Briefly, after cross-linking and sonication, sheared chromatin was immunoprecipitated with an antibody directed against H3K27me3 at a concentration of 1:50 (Cell Signaling Technology) or an equal concentration of normal rabbit IgG (Santa Cruz Biotechnology). Samples were then washed, reverse cross-linked, and proteinase K treated to obtain purified DNA fragments. RT-qPCR was performed using primers specific for a sequence of the mouse Jag1 promoter (Supplemental Table 4).

\section{Statistics}

Data are expressed as the mean \pm SEM. Statistical significance was determined by 1-way ANOVA with a Fisher's least significant difference (LSD) post-hoc test for comparisons of multiple groups and a 2-tailed Student'st test for comparisons between 2 groups (or a KruskalWallis or Mann-Whitney $U$ test for nonparametric data). Statistical analyses were performed using GraphPad Prism 6 for Mac OS $\mathrm{X}$ (GraphPad Software). A $P$ value of less than 0.05 was considered statistically significant.

\section{Study approval}

All experimental procedures for the rodent studies adhered to the guidelines of the Canadian Council on Animal Care and were approved by the St. Michael's Hospital Animal Care Committee. The human study was approved by the Nova Scotia Health Authority Research Ethics Board and the Research Ethics Board of St. Michael's Hospital and was conducted in accordance with Declaration of Helsinki principles. A waiver of consent based on impracticability criteria was provided by the Nova Scotia Health Authority Research Ethics Board.

\section{Author contributions}

SM and KT designed and performed the experiments, analyzed the data, and wrote the manuscript. SNB and TAA performed experiments and analyzed data. BBB and MGK performed in vivo experiments. YL performed in vitro experiments. SLA performed the light microscopic histology studies. KEW performed the electron microscopic studies. LG, KKT, PP, and FSS contributed to the human data presented in the work. AA designed the experiments, supervised the study, and wrote the manuscript.

\section{Acknowledgments}

The authors thank Kryski Biomedia for the artwork. These studies were supported by a Biomedical Research Grant from the Kidney Foundation of Canada and an Operating Grant from Diabetes Canada (OG-3-14-4502-AA) and in part by Grant-inAid funding from the Heart and Stroke Foundation of Canada (G-14-0005877, to AA) and a Canadian Institutes of Health Research Operating Grant (MOP-133631, to AA). SM was supported by a Diabetes Canada Postdoctoral Fellowship. KT was supported by a Research Internship Abroad grant from the Sao Paulo Research Foundation (Fapesp 2016/04591-1). SNB is supported by a Keenan Family Foundation KRESCENT Postdoctoral Fellowship Award and was supported by a Heart and Stroke/Richard Lewar Center of Excellence Fellowship Award and a Banting and Best Diabetes Centre Hugh Sellers Postdoctoral Fellowship. TAA is supported by a King Abdullah Foreign Scholarship. AA is a recipient of a Diabetes Investigator Award from Diabetes Canada. 
Address correspondence to: Andrew Advani, St. Michael's Hospital, 6-151, 61 Queen Street East, Toronto, Ontario, Canada. M5C 2T2. Phone: 416.864.6060, ext.8413; Email: advania@smh.ca.
S. Majumder's present address is: Department of Biological Sciences, Birla Institute of Technology and Sciences (BITS), Pilani, Rajasthan, India.
1. Centers for Disease Control and Prevention. State-specific trends in chronic kidney failure United States, 1990-2001. MMWR Morb Mortal Wkly Rep. 2004;53(39):918-920.

2. Fogo AB. Causes and pathogenesis of focal segmental glomerulosclerosis. Nat Rev Nephrol. 2015;11(2):76-87.

3. Reidy K, Kang HM, Hostetter T, Susztak K. Molecular mechanisms of diabetic kidney disease. JClin Invest. 2014;124(6):2333-2340.

4. Bechtel-Walz W, Huber TB. Chromatin dynamics in kidney development and function. Cell Tissue Res. 2014;356(3):601-608.

5. Conway E, Healy E, Bracken AP. PRC2 mediated H3K27 methylations in cellular identity and cancer. Curr Opin Cell Biol. 2015;37:42-48.

6. Cao R, et al. Role of histone $\mathrm{H} 3$ lysine 27 methylation in Polycomb-group silencing. Science. 2002;298(5595):1039-1043.

7. Trojer P, Reinberg D. Histone lysine demethylases and their impact on epigenetics. Cell. 2006;125(2):213-217.

8. Agger K, et al. UTX and JMJD3 are histone H3K27 demethylases involved in HOX gene regulation and development. Nature. 2007;449(7163):731-734.

9. De Santa F, Totaro MG, Prosperini E, Notarbartolo S, Testa G, Natoli G. The histone H3 lysine-27 demethylase Jmjd3 links inflammation to inhibition of polycomb-mediated gene silencing. Cell. 2007;130(6):1083-1094.

10. Lee MG, et al. Demethylation of H3K27 regulates polycomb recruitment and $\mathrm{H} 2 \mathrm{~A}$ ubiquitination. Science. 2007;318(5849):447-450.

11. Lan F, et al. A histone H3 lysine 27 demethylase regulates animal posterior development. Nature. 2007;449(7163):689-694.

12. Tsukada Y, et al. Histone demethylation by a family of JmjC domain-containing proteins. Nature. 2006;439(7078):811-816.

13. Hong S, Cho YW, Yu LR, Yu H, Veenstra TD, Ge K. Identification of JmjC domain-containing UTX and JMJD3 as histone H3 lysine 27 demethylases. Proc Natl Acad Sci U S A. 2007;104(47):18439-18444.

14. Kim E, Song JJ. Diverse ways to be specific: a novel Zn-binding domain confers substrate specificity to UTX/KDM6A histone H3 Lys 27 demethylase. Genes Dev. 2011;25(21):2223-2226.

15. Sengoku T, Yokoyama S. Structural basis for histone H3 Lys 27 demethylation by UTX/KDM6A. Genes Dev. 2011;25(21):2266-2277.

16. Siddiqi FS, et al. The histone methyltransferase enzyme enhancer of zeste homolog 2 protects against podocyte oxidative stress and renal injury in diabetes. JAm Soc Nephrol. 2016;27(7):2021-2034.

17. Pan M, et al. Regional glutamine deficiency in tumours promotes dedifferentiation through inhibition of histone demethylation. Nat Cell Biol. 2016;18(10):1090-1101.

18. Edeling M, Ragi G, Huang S, Pavenstadt H, Susztak K. Developmental signalling pathways in renal fibrosis: the roles of Notch, Wnt and Hedgehog. Nat Rev Nephrol. 2016;12(7):426-439.

19. Moeller MJ, Sanden SK, Soofi A, Wiggins RC, Holzman LB. Podocyte-specific expression of cre recombinase in transgenic mice. Genesis. 2003;35(1):39-42.

20. Su IH, et al. Ezh2 controls B cell development through histone $\mathrm{H} 3$ methylation and Igh rearrangement. Nat Immunol. 2003;4(2):124-131.

21. D'Agati VD, Kaskel FJ, Falk RJ. Focal segmental glomerulosclerosis. $N$ Engl J Med. 2011;365(25):2398-2411.

22. MMRC. Strain Certification: A service of the UNC Mutant Mouse Regional Resource Center. NIH Website. http://www.csbio.unc.edu/ MMRRC/index.py?run=StatsTable. viewSample \&sample $=1538 \& \mathrm{~mm}=0$ Accessed November 14, 2017.

23. Arif E, Solanki AK, Nihalani D. Adriamycin susceptibility among C57BL/6 substrains. Kidney Int. 2016;89(3):721-723.

24. Niranjan T, et al. The Notch pathway in podocytes plays a role in the development of glomerular disease. Nat Med. 2008;14(3):290-298.

25. Sweetwyne MT, Gruenwald A, Niranjan T, Nishinakamura R, Strobl LJ, Susztak K. Notch1 and Notch2 in podocytes play differential roles during diabetic nephropathy development. Diabetes. 2015;64(12):4099-4111.

26. Fogo AB. Animal models of FSGS: lessons for pathogenesis and treatment. Semin Nephrol. 2003;23(2):161-171.

27. Knutson SK, et al. Durable tumor regression in genetically altered malignant rhabdoid tumors by inhibition of methyltransferase EZH2. Proc Natl Acad Sci U S A. 2013;110(19):7922-7927.

28. Cheng HT, Miner JH, Lin M, Tansey MG, Roth K, Kopan R. Gamma-secretase activity is dispensable for mesenchyme-to-epithelium transition but required for podocyte and proximal tubule formation in developing mouse kidney. Development. 2003;130(20):5031-5042.

29. Murea M, et al. Expression of Notch pathway proteins correlates with albuminuria, glomerulosclerosis, and renal function. Kidney Int. 2010;78(5):514-522.

30. Kruidenier L, et al. A selective jumonji H3K27 demethylase inhibitor modulates the proinflammatory macrophage response. Nature. 2012;488(7411):404-408.

31. Herman-Edelstein M, Thomas MC, Thallas-Bonke V, Saleem M, Cooper ME, Kantharidis P. Dedifferentiation of immortalized human podocytes in response to transforming growth factor- $\beta$ : a model for diabetic podocytopathy. Diabetes. 2011;60(6):1779-1788.

32. Heinemann $B$, et al. Inhibition of demethylases by GSK-J1/J4. Nature. 2014;514(7520):E1-E2.

33. Saleem MA, et al. A conditionally immortalized human podocyte cell line demonstrating nephrin and podocin expression. J Am Soc Nephrol. 2002;13(3):630-638.
34. Jaenisch R, Bird A. Epigenetic regulation of gene expression: how the genome integrates intrinsic and environmental signals. Nat Genet. 2003;33(suppl):245-254.

35. O'Carroll D, Erhardt S, Pagani M, Barton SC, Surani MA, Jenuwein T. The polycomb-group gene Ezh2 is required for early mouse development. Mol Cell Biol. 2001;21(13):4330-4336.

36. Varambally $S$, et al. The polycomb group protein EZH2 is involved in progression of prostate cancer. Nature. 2002;419(6907):624-629.

37. Lefevre GM, Patel SR, Kim D, Tessarollo L, Dressler GR. Altering a histone H3K4 methylation pathway in glomerular podocytes promotes a chronic disease phenotype. PLoS Genet. 2010;6(10):e1001142.

38. Isermann $\mathrm{B}$, et al. Activated protein $\mathrm{C}$ protects against diabetic nephropathy by inhibiting endothelial and podocyte apoptosis. Nat Med. 2007;13(11):1349-1358.

39. Hasegawa K, et al. Renal tubular Sirt1 attenuates diabetic albuminuria by epigenetically suppressing Claudin-1 overexpression in podocytes. Nat Med. 2013;19(11):1496-1504.

40. Lin CL, et al. MicroRNA-29a promotion of nephrin acetylation ameliorates hyperglycemiainduced podocyte dysfunction. J Am Soc Nephrol. 2014;25(8):1698-1709.

41. Schwanbeck R. The role of epigenetic mechanisms in Notch signaling during development. JCell Physiol. 2015;230(5):969-981.

42. Arcipowski KM, Martinez CA, Ntziachristos P. Histone demethylases in physiology and cancer: a tale of two enzymes, JMJD3 and UTX. Curr Opin Genet Dev. 2016;36:59-67.

43. Zha L, et al. The histone demethylase UTX promotes brown adipocyte thermogenic program via coordinated regulation of $\mathrm{H} 3 \mathrm{~K} 27$ demethylation and acetylation. J Biol Chem. 2015;290(41):25151-25163.

44. Ohtani K, et al. Epigenetic regulation of endothelial lineage committed genes in pro-angiogenic hematopoietic and endothelial progenitor cells. Circ Res. 2011;109(11):1219-1229.

45. Greenfield A, et al. The UTX gene escapes X inactivation in mice and humans. Hum Mol Genet. 1998;7(4):737-742.

46. Xu J, Deng X, Watkins R, Disteche CM. Sexspecific differences in expression of histone demethylases Utx and Uty in mouse brain and neurons. JNeurosci. 2008;28(17):4521-4527.

47. Ntziachristos $\mathrm{P}$, et al. Contrasting roles of histone 3 lysine 27 demethylases in acute lymphoblastic leukaemia. Nature. 2014;514(7523):513-517.

48. Miller SA, Mohn SE, Weinmann AS. Jmjd3 and UTX play a demethylase-independent role in chromatin remodeling to regulate T-box family member-dependent gene expression. Mol Cell. 2010;40(4):594-605.

49. Berthier CC, et al. Enhanced expression of Janus kinase-signal transducer and activator of transcription pathway members in human diabetic 
nephropathy. Diabetes. 2009;58(2):469-477.

50. Margueron R, et al. Ezh1 and Ezh2 maintain repressive chromatin through different mechanisms. Mol Cell. 2008;32(4):503-518.

51. Felician G, et al. Epigenetic modification at Notch responsive promoters blunts efficacy of inducing notch pathway reactivation after myocardial infarction. Circ Res. 2014;115(7):636-649.

52. Zhao E, et al. Cancer mediates effector $\mathrm{T}$ cell dysfunction by targeting microRNAs and EZH2 via glycolysis restriction. Nat Immunol. 2016;17(1):95-103.

53. Dai C, Stolz DB, Kiss LP, Monga SP, Holzman LB, Liu Y. Wnt/beta-catenin signaling promotes podocyte dysfunction and albuminuria. J Am Soc Nephrol. 2009;20(9):1997-2008.

54. Koyanagi M, Bushoven P, Iwasaki M, Urbich C, Zeiher AM, Dimmeler S. Notch signaling contributes to the expression of cardiac markers in human circulating progenitor cells. Circ Res. 2007;101(11):1139-1145.

55. Lako M, Strachan T, Bullen P, Wilson DI, Robson SC, Lindsay S. Isolation, characterisation and embryonic expression of WNT11, a gene which maps to 11q13.5 and has possible roles in the development of skeleton, kidney and lung. Gene. 1998;219(1-2):101-110.

56. Zhang P, Cai Y, Soofi A, Dressler GR. Activation of Wnt11 by transforming growth factor- $\beta$ drives mesenchymal gene expression through non- canonical Wnt protein signaling in renal epithelial cells. J Biol Chem. 2012;287(25):21290-21302.

57. Alghamdi TA, et al. Janus Kinase 2 regulates transcription factor EB expression and autophagy completion in glomerular podocytes. J Am Soc Nephrol. 2017;28(9):2641-2653.

58. Jeansson M, Björck K, Tenstad O, Haraldsson B. Adriamycin alters glomerular endothelium to induce proteinuria. J Am Soc Nephrol. 2009;20(1):114-122.

59. Zhao ZL, et al. NOTCH1 inhibition enhances the efficacy of conventional chemotherapeutic agents by targeting head neck cancer stem cell. Sci Rep. 2016;6:24704.

60. Daehn I, et al. Endothelial mitochondrial oxidative stress determines podocyte depletion in segmental glomerulosclerosis. J Clin Invest. 2014;124(4):1608-1621.

61. Knutson SK, et al. Selective inhibition of EZH2 by EPZ-6438 leads to potent antitumor activity in EZH2-mutant non-Hodgkin lymphoma. Mol Cancer Ther. 2014;13(4):842-854.

62. Advani A, et al. Fluorescent microangiography is a novel and widely applicable technique for delineating the renal microvasculature. PLOS One. 2011;6(10):e24695.

63. Schindelin J, et al. Fiji: an open-source platform for biological-image analysis. Nat Methods. 2012;9(7):676-682.

64. Schneider CA, Rasband WS, Eliceiri KW. NIH
Image to ImageJ: 25 years of image analysis. Nat Methods. 2012;9(7):671-675.

65. Shankland SJ, Pippin JW, Couser WG. Complement (C5b-9) induces glomerular epithelial cell DNA synthesis but not proliferation in vitro. Kidney Int. 1999;56(2):538-548.

66. Stitt-Cavanagh EM, et al. A maladaptive role for EP4 receptors in podocytes. J Am Soc Nephrol. 2010;21(10):1678-1690.

67. Weibel ER, Gomez DM. A principle for counting tissue structures on random sections. J Appl Physiol. 1962;17:343-348.

68. Batchu SN, et al. Prostaglandin I2 receptor agonism preserves $\beta$-cell function and attenuates albuminuria through nephrin-dependent mechanisms. Diabetes. 2016;65(5):1398-1409.

69. Endlich N, et al. Podocytes respond to mechanical stress in vitro. J Am Soc Nephrol. 2001;12(3):413-422.

70. Qi W, et al. An allosteric PRC2 inhibitor targeting the H3K27me 3 binding pocket of EED. Nat Chem Biol. 2017;13(4):381-388.

71. Benyoucef A, et al. UTX inhibition as selective epigenetic therapy against TAL1-driven T-cell acute lymphoblastic leukemia. Genes Dev. 2016;30(5):508-521.

72. Majumder S, et al. G-protein-coupled receptor-2-interacting protein-1 controls stalk cell fate by inhibiting $\Delta$-like 4 -Notch1 signaling. Cell Rep . 2016;17(10):2532-2541. 